\title{
ON THE ACCURATE LONG-TIME SOLUTION OF THE WAVE EQUATION IN EXTERIOR DOMAINS: ASYMPTOTIC EXPANSIONS AND CORRECTED BOUNDARY CONDITIONS
}

\author{
THOMAS HAGSTROM, S. I. HARIHARAN, AND R. C. MACCAMY
}

\begin{abstract}
We consider the solution of scattering problems for the wave equation using approximate boundary conditions at artificial boundaries. These conditions are explicitly viewed as approximations to an exact boundary condition satisfied by the solution on the unbounded domain. We study both the shortand long-time behavior of the error. It is proved that, in two space dimensions, no local in time, constant-coefficient boundary operator can lead to accurate results uniformly in time for the class of problems we consider. A variablecoefficient operator is developed which attains better accuracy (uniformly in time) than is possible with constant-coefficient approximations. The theory is illustrated by numerical examples. We also analyze the proposed boundary conditions, using energy methods and leading to asymptotically correct error bounds.
\end{abstract}

\section{INTRODUCTION}

Problems posed on unbounded spatial domains arise naturally in the study of wave propagation. The standard approach to the numerical solution of such problems is to introduce an artificial boundary and apply 'appropriate' boundary conditions. A vast literature has appeared in the past 15 years, devoted primarily to the derivation of boundary conditions for the wave equation. (See [10] for a recent review.) Surprisingly, one finds very little precise error analysis in this body of work. Notable exceptions to this are the paper of Halpern and Rauch [17], where error estimates in terms of the reflection coefficient are given, and the early work of Bayliss and Turkel [3], who give estimates based on the size of the computational domain. In both cases the error analysis is (generally) not uniform in time. In the first case, this is manifested in the requirement that the solution not be too smooth, while in the second it is related to the long-time breakdown of the progressive wave expansion.

In this work we consider both the short- and long-time error for finite domain approximations to limiting amplitude problems for the wave equation in

Received by the editor October 27, 1992 and, in revised form, September 21, 1993.

1991 Mathematics Subject Classification. Primary 65M99, 35B40.

Key words and phrases. Boundary conditions, asymptotic expansions, wave equation.

The first author supported in part by NSF Grants DMS-8905314, DMS-9108072, and DOE Grant DE-FG03-92ER25128, the second supported in part by NSF Grant DMS-8921189 and the third by NSF Grant DMS-9001012. The first and second authors were also partially supported by ICOMP, NASA-Lewis Research Center. 
exterior domains. By limiting amplitude problems we mean cases where the forcing becomes steady or time-periodic as $t \rightarrow \infty$, and one wishes to accurately compute the solution as it approaches a steady or time-periodic state. (In contrast, Engquist and Halpern [7, 8] consider mainly the rate of decay of the approximate solution to steady-state and are not interested in accurately reproducing the transient behavior of the actual solution.) We concentrate on what turns out to be the most difficult case: zero frequency (asymptotically steady) and two space dimensions. However, we do give some discussion of the other problems.

In $\S 2$ we develop an asymptotic error analysis for general local in time constant-coefficient boundary conditions at a circular artificial boundary in two space dimensions. (We note that most of the boundary conditions which have been proposed fall into the category of local in time with constant coefficients.) Conditions are analyzed as approximations to the exact boundary operator, which can be conveniently expressed at such boundaries. It is seen that the behavior of the exact operator for low frequencies cannot be approximated by operators in the class considered. This immediately leads to lower bounds on the error of the order $\frac{1}{\ln t}$ for long times. These are independent of the coefficients in the boundary condition and of the size of the computational domain.

In $\S 3$ we show how to add variable-coefficient corrections to the boundary conditions, which allow us to beat the lower bounds derived earlier. We present the modified conditions only for the case of a circular artificial boundary, though we do make some remarks in the appendix on their extension to general boundaries. All rigorous error analyses are given for the case of a circular inner boundary. We believe the basic results will hold for an arbitrary star-shaped scatterer, but we do not have complete proofs. These results are illustrated by numerical experiments in $\S 4$. In particular, for a very simple test case, we find that for all of the standard conditions tried, the long-time error exceeds $5 \%$. The use of the corrected condition results in an order of magnitude improvement.

Section 5 is devoted to the analysis of the proposed conditions for computations in the annular region $1<r<R$. Using a somewhat involved sequence of energy estimates, we prove that for long times the error is of the order $R^{-1 / 2}(\ln R)^{2}(\ln t)^{-3}$. This shows that the error does in fact decay more rapidly than the $(\ln t)^{-1}$ lower bound of the constant-coefficient case. Moreover, we show that the major source of error resides in the radially symmetric component. If this component is subtracted out, then the error becomes $O\left((\ln R)^{1 / 2} \ln t \cdot t^{-5 / 2}\right)$. Short-time error estimates follow from the arguments of [3]. (For an energy analysis of the 'standard' boundary conditions, see HaDuong and Joly [13].) We note that the method could be extended to improve the long-time error estimates by simply using a better approximation to the function $\mathscr{H}$ defined in the appendix.

In our work we normalize the wave equation $c^{-2} u_{t t}=\nabla^{2} u$ to have $c=1$. To obtain the general case, one needs only replace $t$ by $c t$. It is interesting to note that in the electromagnetic problems at moderate frequencies $c$ is very large (of the order $10^{9}$ ), so that our 'large $t$ ' estimates essentially always hold and the improvement from $(\ln t)^{-1}$ to $(\ln t)^{-3}$ is very significant.

Some of the results presented here, as well as a more general discussion of the error analysis of approximate boundary conditions, can be found in [14]. 
Some auxiliary computations, including the construction and approximation of a special function which plays an important role in the analysis, as well as extensions to three dimensions and noncircular boundaries, can be found in the appendices. These are included in the supplement section at the end of the issue.

\section{ASYMPTOTIC ERROR ANALYSIS}

Preliminary to our study of the long-time behavior of the error which results from truncating the domain, we recall the basic theorems on the long-time behavior of solutions in exterior domains (with convex or star-shaped boundaries). We consider three distinct cases: the Cauchy problem with compactly supported data, asymptotically constant forcing, and asymptotically time-periodic forcing. In the first case the solution decays to zero, while in the others (under appropriate assumptions) it approaches steady or periodic states, the so-called principle of limiting amplitude. In three space dimensions, the transient behavior typically decays exponentially in time [22,24]. In two dimensions, in contrast, the transient behavior can be quite persistent. In an early paper, Chen [5] quantified this for a plane pulse incident on a circular cylinder. General theorems on the decay of the transients have since been given by Muravei [25], and are summarized in Table 1.

The approximation of exterior problems on bounded domains leads to further questions concerning long-time behavior. In particular,

(i) Does the approximate solution approach a steady (time-periodic) state?

(ii) What is the error in the final state?

(iii) What is the transient behavior of the error?

An affirmative answer to the first question seems a reasonable requirement to make on any approximation scheme, and is closely related to the notion of dissipativity for boundary conditions as introduced by Barry, Bielak, and MacCamy $[2,4]$. By concentrating on convergence to steady state, it is possible to make the final error small or zero by choosing boundary conditions which reduce to asymptotic or exact conditions for the Poisson equation. (See $[7,8,16]$.) It is on the third question that we focus our attention here. In particular, we will derive lower bounds of the form $\frac{\ln r}{\ln t}$ for the error due to domain truncation and the imposition of any constant-coefficient, local in time boundary condition for a two-dimensional model problem. That is, no approximation of this type can accurately simulate the transient behavior associated with convergence to steady state on domains of reasonable size. In contrast, problems in three dimensions can be accurately solved using such 'standard' boundary operators.

TABLE 1. Decay of transients in 2d from Muravei [25]

\begin{tabular}{|r||c|c|c|}
\hline Boundary Condition & Cauchy & $f \rightarrow f_{\infty}(x, y)$ & $f \rightarrow f_{\infty}(x, y) \cdot \sin (\omega t+\phi)$ \\
\hline \hline Dirichlet or Mixed & $O\left(\frac{1}{t \ln ^{2} t}\right)$ & $O\left(\frac{1}{\ln t}\right)$ & $O\left(\frac{1}{\omega t \ln ^{2} t}\right)$ \\
\hline Neumann or Impedance & $O\left(\frac{1}{t}\right)$ & May not converge & $O\left(\frac{1}{\omega t}\right)$ \\
\hline
\end{tabular}


2.1. Properties of the exact condition and common approximations. Consider the wave equation forced at the surface of a cylinder:

$$
\begin{gathered}
\frac{\partial^{2} u}{\partial t^{2}}=\frac{\partial^{2} u}{\partial r^{2}}+\frac{1}{r} \frac{\partial u}{\partial r}+\frac{1}{r^{2}} \frac{\partial^{2} u}{\partial \theta^{2}}, \quad r>1, \\
u(r, \theta, 0)=\frac{\partial u}{\partial t}(r, \theta, 0)=0, \\
u(1, \theta, t)=g(\theta, t)=g_{\infty}(\theta)+O\left(\frac{1}{t^{2}}\right), t \rightarrow \infty .
\end{gathered}
$$

From Muravei [25], as $t \rightarrow \infty, u=u_{\infty}(r, \theta)+O\left(\frac{1}{\ln t}\right)$, where $u_{\infty}$ is the solution of Laplace's equation exterior to the cylinder satisfying the inhomogeneous Dirichlet condition, $u_{\infty}(1, \theta)=g_{\infty}(\theta)$. It is convenient to express $u$ using a Fourier series in $\theta$ and Laplace transforms in $t$. Let

$$
\begin{aligned}
\hat{u}_{n}(r, s) & =\frac{1}{2 \pi} \int_{0}^{\infty} e^{-s t} \int_{0}^{2 \pi} e^{-i n \theta} u(r, \theta, t) d \theta d t \\
\hat{g}_{n}(s) & =\frac{1}{2 \pi} \int_{0}^{\infty} e^{-s t} \int_{0}^{2 \pi} e^{-i n \theta} g(\theta, t) d \theta d t
\end{aligned}
$$

We then have

$$
u=\Re\left(\sum_{n=0}^{\infty} e^{i n \theta} \mathscr{L}^{-1}\left(\hat{u}_{n}(r, s)\right)\right)
$$

where

$$
\hat{u}_{n}=\left(\frac{K_{n}(r s)}{K_{n}(s)}\right) g_{n}(s) .
$$

Here, $\Re$ denotes the real part, $\mathscr{L}^{-1}$ denotes the inverse Laplace transform, and $K_{n}$ (and later $I_{n}$ ) are the modified Bessel functions [1].

To approximate $u$ by the solution, $v$, to a problem on a bounded domain, introduce an artificial boundary at $r=R$. A general form for a local in time, constant-coefficient boundary condition is

$$
\frac{\partial \hat{v}_{n}}{\partial r}=-b_{n}(s) \hat{v}_{n}
$$

where $b_{n}$ is a rational function of $s$ with real coefficients. If, in addition, locality in space is required, then $b_{n}$ is also constrained to be a real rational function of $i \cdot n$. Note that conditions involving higher-order $r$-derivatives may be put in this form by substituting $\left(s^{2}-\frac{1}{r} \frac{\partial}{\partial r}+\frac{n^{2}}{r^{2}}\right)$ for $\frac{\partial^{2}}{\partial r^{2}}$.

We view (2.1.7) as an approximation to the exact boundary condition at $r=R$ :

$$
\frac{\partial \hat{u}_{n}}{\partial r}=\left(\frac{s K_{n}^{\prime}(R s)}{K_{n}(R s)}\right) \hat{u}_{n} \equiv-b_{n}^{e}(s) \hat{u}_{n}
$$


This condition, of course, is nonlocal in space and time. The error, $e=u-v$, is easily expressed in transform space. (See also [14].) It satisfies

$$
\frac{d^{2} \hat{e}_{n}}{d r^{2}}+\frac{1}{r} \frac{d \hat{e}_{n}}{d r}-\frac{n^{2}}{r^{2}} \hat{e}_{n}=0, \quad 1<r<R,
$$

$$
\hat{e}_{n}(1)=0, \quad\left(\frac{d \hat{e}_{n}}{d r}(R)+b_{n}(s) \hat{e}_{n}(R)\right)=\left(b_{n}(s)-b_{n}^{e}(s)\right) \hat{u}_{n}(R, s) .
$$

Solving this yields

$$
\hat{e}_{n}(r, s)=A(R, s) \cdot\left[\frac{K_{n}(r s)}{K_{n}(s)}-\frac{I_{n}(r s)}{I_{n}(s)}\right] \cdot g_{n}(s) \frac{K_{n}(R s)}{K_{n}(s)},
$$

where $A(R, s)$, which is the term we control by our choice of boundary condition, is given by

$$
A(R, s)=\left(b_{n}(s)-b_{n}^{e}(s)\right) \cdot B(R, s),
$$

$$
B(R, s)=\left(\left(b_{n}(s)-b_{n}^{e}(s)\right) \frac{K_{n}(R s)}{K_{n}(s)}-\left(\frac{s I_{n}^{\prime}(R s)}{I_{n}(R s)}+b_{n}(s)\right) \frac{I_{n}(R s)}{I_{n}(s)}\right)^{-1} .
$$

In order to analyze the approximation properties of $b_{n}(s)$, we need to study the behavior of $b_{n}^{e}(s)$. For short to moderate times, and large domains, we consider the limit $|R s| \equiv|z| \gg 1$. Using standard results on the asymptotic behavior of modified Bessel functions (e.g., [1]), we find, as $z \rightarrow \infty$,

$$
b_{n}^{e}(s)=-\frac{1}{R} \frac{z K_{n}^{\prime}(z)}{K_{n}(z)}=\frac{1}{R}\left(z+\frac{1}{2}+\frac{\left(4 n^{2}-1\right)}{8 z}-\frac{\left(4 n^{2}-1\right)}{8 z^{2}}+O\left(z^{-3}\right)\right) .
$$

This expansion is closely related to Friedlander's progressive wave expansion used to construct boundary conditions in $[3,15]$. Essentially all boundary conditions proposed in the literature agree with at least the first term.

We expect, on the other hand, that the long-time behavior of solutions converging to steady state will be governed by the behavior of the transform near $s=0$. (This corresponds to the nonuniformity in time of the progressive wave expansion.) The relevant expansion of $b_{n}^{e}$ is

$$
b_{n}^{e}(s)= \begin{cases}\frac{-1}{R\left(\ln \left(\frac{z}{2}\right)+\gamma\right)}+O\left(z^{2}\right), & n=0, \\ \frac{n}{R}+O\left(z^{2}\right), & n \neq 0 .\end{cases}
$$

Matching this expansion introduces more difficulties than matching (2.1.14). If we simply insist that $b_{n}(0)=b_{n}^{e}(0)$, we see that $b_{n}$ must be a function of $n$ rather than $i \cdot n$. This means that the boundary operator must be nonlocal in $\theta$. Moreover, we cannot match the $\frac{1}{\ln s}$ behavior for $n=0$ with a rational function of $s$. We shall see below that this fact practically eliminates the possibility of accurate long-time simulations using time-local boundary conditions with constant coefficients. 
It is of interest to recast into the present form various boundary conditions which have appeared in the literature, and to study them in the large- and small$s$ limits. In a later section we will see how these properties are reflected in their performance. One should also keep in mind the concept of dissipativity discussed in $[2,4]$ : a boundary condition for the wave equation is called dissipative if it excludes poles in the closed right-half transform plane except at $s=0$. We would extend this to admit only simple poles at $s=0$. These conditions are necessary if solutions are to remain bounded as $t \rightarrow \infty$.

Engquist and Majda [6], Bayliss and Turkel [3]:

$$
\frac{\partial v}{\partial t}+\frac{\partial v}{\partial r}+\frac{v}{2 R}=0
$$

$$
b_{n}(s)=\frac{1}{R}\left(z+\frac{1}{2}\right) \text {, }
$$

Bayliss and Turkel (2nd order) [3]:

$$
b_{n}-b_{n}^{e}= \begin{cases}O\left(z^{-1}\right), & z \rightarrow \infty \\ -\frac{2 n-1}{2 R}+O\left(\ln ^{-1} z\right), & z \rightarrow 0 .\end{cases}
$$

$$
\begin{gathered}
\left.\prod_{j=1}^{2}\left(\frac{\partial}{\partial t}+\frac{\partial}{\partial r}+\frac{4 j-3}{2 r}\right) v\right|_{r=R}=0 \\
b_{n}(s)=\frac{1}{R} \frac{\left(z^{2}+\frac{3}{2} z+\frac{4 n^{2}+3}{8}\right)}{z+1}
\end{gathered}
$$

$$
b_{n}-b_{n}^{e}= \begin{cases}O\left(z^{-3}\right), & z \rightarrow \infty, \\ \frac{4 n^{2}-8 n+3}{8 R}+O\left(\ln ^{-1} z\right), & z \rightarrow 0 .\end{cases}
$$

Engquist and Majda (3rd order) [6]:

$$
\begin{gathered}
\frac{\partial^{3} v}{\partial r \partial t^{2}}+\frac{\partial^{3} v}{\partial t^{3}}-\frac{1}{2 R^{2}} \frac{\partial^{3} v}{\partial t \partial \theta^{2}}+\frac{1}{2 R} \frac{\partial^{2} v}{\partial t^{2}}+\frac{1}{2 R^{3}} \frac{\partial^{2} v}{\partial \theta^{2}}=0 \\
b_{n}(s)=\frac{1}{R} \frac{\left(z^{3}+\frac{1}{2} z^{2}+\frac{n^{2}}{2} z-\frac{n^{2}}{2}\right)}{z^{2}}, \\
b_{n}-b_{n}^{e}= \begin{cases}O\left(z^{-1}\right), & z \rightarrow \infty \\
-\frac{n^{2}}{2 R z^{2}}, & z \rightarrow 0\end{cases}
\end{gathered}
$$

Engquist and Halpern [7]:

$$
\begin{gathered}
\frac{\partial v}{\partial t}+\frac{\partial v}{\partial r}+K v=0 \\
b_{n}(s)=\frac{1}{R}(z+n)
\end{gathered}
$$




$$
b_{n}-b_{n}^{e}= \begin{cases}\frac{1}{2 R}, & z \rightarrow \infty \\ O\left(\ln ^{-1} z\right), & z \rightarrow 0\end{cases}
$$

(Here, $K$ is simply the Dirichlet-to-Neumann map for the exterior Laplace equation.) Higdon [19] has proposed simple product boundary conditions for plane boundaries. It is interesting to study their naive generalization to the circular case (we emphasize that these are not advocated in [19]):

$$
\begin{aligned}
& \prod_{j=1}^{2}\left(c_{j} \frac{\partial}{\partial t}+\frac{\partial}{\partial r}\right) v=0 \\
& b_{n}(s)=\frac{1}{R} \frac{\left(1+c_{1} c_{2}\right) z^{2}}{\left(c_{1}+c_{2}\right) z-1}
\end{aligned}
$$

$$
b_{n}-b_{n}^{e}= \begin{cases}\frac{\left(1+c_{1} c_{2}-c_{1}-c_{2}\right)}{c_{1}+c_{2}} z+\frac{2-c_{1}^{2}-c_{2}^{2}}{2\left(c_{1}+c_{2}\right)^{2}}+O\left(z^{-1}\right), & z \rightarrow \infty \\ -n+O\left(\ln ^{-1} z\right), & z \rightarrow 0\end{cases}
$$

We see that condition (2.1.19) is the most accurate in the large- $z$ limit, while (2.1.25) is the least. Condition (2.1.25), on the other hand, is the only one with the correct $z=0$ limit (and, hence, is nonlocal in space). This corresponds to the fact that it was designed for steady-state calculations. It is surprising that (2.1.22) matches no more terms in the large- $z$ expansion than (2.1.16). Note, however, that it does approximate them in the large- $n$ limit. Condition (2.1.28) can be made to match the first two terms for large $z$ by making $c_{1}=c_{2}=1$.

Conditions $(2.1 .22,2.1 .28)$ are clearly not dissipative. In fact, our expression for the Laplace transform of (2.1.28) displays a singularity in the right-half transform plane, indicating exponential growth of the solution and, hence, the error. (This will be confirmed in the numerical experiments.) The singularity in the transform of (2.1.22) is at $s=0$. This allows algebraic growth in time. For example, it may be verified that $t \ln r$ satisfies the wave equation, a homogeneous Dirichlet condition at $r=1$, as well as (2.1.22). Therefore, these boundary conditions are inappropriate for long-time calculations. This conclusion echoes that of Gustafsson [12], who considered channel-like domains.

2.2. Long-time asymptotics. All the dissipative conditions above, excepting (2.1.25), will not result in the correct steady-state limit. Moreover, no local in time constant-coefficient boundary condition can have $b_{0}(s)$ behaving correctly near $s=0$. By studying the $s \rightarrow 0$ limit of (2.1.11), we can develop candidate asymptotic expansions of the error as $t \rightarrow \infty$ for general rational approximations. Although the actual behavior of the error can be worse than these candidate expansions predict (as with nondissipative conditions), standard Tauberian theorems show that it can never be better.

The small-s behavior of each term in (2.1.11) is easily computed. We begin with the cases $n \neq 0$, which are simplest:

$$
\hat{e}_{n}(r, s)=\left(\frac{\left(r^{n}-r^{-n}\right)\left(\frac{n}{R}-b_{n}(0)\right)}{R^{n}\left(\frac{n}{R}+b_{n}(0)\right)-R^{-n}\left(\frac{n}{R}-b_{n}(0)\right)}\right) R^{-n} \frac{g_{\infty, n}}{s}+O(1),
$$


which suggests, as $t \rightarrow \infty$,

$$
e_{n}(r, t)=\left(\frac{\left(r^{n}-r^{-n}\right)\left(\frac{n}{R}-b_{n}(0)\right)}{R^{n}\left(\frac{n}{R}+b_{n}(0)\right)-R^{-n}\left(\frac{n}{R}-b_{n}(0)\right)}\right) R^{-n} g_{\infty, n}+o\left(t^{-1}\right) .
$$

We note that $(2.2 .32)$ generally will hold if the solution has a limit. The limiting value of the error is simply the error in approximating the Poisson equation with the boundary conditions defined by $b_{n}(0)$. It is zero if $b_{n}(0)=\frac{n}{R}$. As the function in parentheses is bounded by 1 , we have in general that the maximum of $e_{n}(r, \infty)$ is of the size $R^{-n}$ and decays algebraically off the artificial boundary. Practically, this means that only the small- $n$ harmonics need be correctly treated for $s=0$.

For $n=0$ we have

$$
\hat{e}_{0}(r, s)=\left(\frac{b_{0}(0)}{1+b_{0}(0) \ln R}+\frac{1}{\ln \frac{s}{2}+\gamma}\right) \ln r \cdot \frac{g_{\infty, 0}}{s}+O(1) .
$$

This is consistent with the $t \rightarrow \infty$ expansion

$$
e_{0}(r, t)=\left(\frac{b_{0}(0)}{1+b_{0}(0) \ln R}+\mathscr{H}\left(2 e^{-\gamma} t\right)\right) \ln r \cdot g_{\infty, 0}+o\left(t^{-1}\right),
$$

where, $\mathscr{H}(\tau)$ is a function whose Laplace transform behaves like $(s \ln s)^{-1}$ as $s \rightarrow 0, O\left((s \ln s)^{-1}\right)$ as $s \rightarrow \infty$ and has no other singularities in the closed right half-plane. It is given by

$$
\mathscr{H}(\tau)=-\int_{\tau}^{\infty} e^{\tau-p}\left(\int_{0}^{1} \frac{p^{u-1}}{\Gamma(u)} d u\right) d p .
$$

Its asymptotic behavior is

$$
\begin{aligned}
\mathscr{H}(\tau) & =-\frac{1}{\ln \tau}+\frac{\gamma}{(\ln \tau)^{2}}+O\left((\ln \tau)^{-3}\right) \\
& =-\frac{1}{\ln \left(\tau e^{\gamma}\right)}+O\left((\ln \tau)^{-3}\right), \quad \tau \rightarrow \infty .
\end{aligned}
$$

(The construction of $\mathscr{H}$ and its asymptotic expansion are given in Appendix A.)

Note the very slow decay of this error with $R, r$, and $t$. In particular, it may not be detected by varying $R$, for $R$ large. Also note that the second term in the expansion is independent of the boundary condition. Therefore, it will be present for any dissipative condition, and hence provides a lower bound on the attainable long-time accuracy for local in time, constant-coefficient operators. This fact follows from

Theorem 1. Let $e=u-v$ be the error resulting from domain truncation with the boundary condition (2.1.7) and let $e_{0}(r, t)=\frac{1}{2 \pi} \int_{0}^{2 \pi} e(r, \theta, t) d \theta$. Suppose $\lim _{t \rightarrow \infty} e_{0}(r, t)$ exists. Then

$$
\underset{t \rightarrow \infty}{\limsup } \ln t \cdot\left|e_{0}(r, t)-e_{0}(r, \infty)\right| \geq \ln r \cdot\left|g_{\infty, 0}\right|
$$


Proof. The proof follows from the Hardy-Littlewood-Karamata Theorem [28, §.1, Theorem 3]. First of all, by the Abelian theorems for Laplace transforms (e.g., $[29, \S 5.1])$,

$$
e_{0}(r, \infty)=\left(\frac{b_{0}(0)}{1+b_{0}(0) \ln R}\right) \ln r \cdot g_{\infty, 0} .
$$

Let $w(r, t)= \pm\left(e_{0}(r, \infty)-e_{0}(r, t)\right)$. Then $\hat{w}(r, s)$ is given by \pm the second term in (2.2.33) as $s \rightarrow 0$. Suppose (2.2.37) is false. Then for some $K>0$, $\tilde{w} \equiv w+\frac{K}{\ln t}$ is eventually positive. Moreover,

$$
\hat{\tilde{w}}(r, s) \sim-\frac{\ln r \cdot\left|g_{\infty}, 0\right|+K}{s \ln s}, s \rightarrow 0 .
$$

The Hardy-Littlewood-Karamata Theorem then implies

$$
\frac{\ln t}{t} \int_{t_{0}}^{t} \tilde{w}(r, p) d p \rightarrow \ln r \cdot\left|g_{\infty}, 0\right|+K, \quad t \rightarrow \infty,
$$

which implies

$$
\frac{\ln t}{t} \int_{t_{0}}^{t} w(r, p) d p \rightarrow \ln r \cdot\left|g_{\infty, 0}\right| .
$$

This contradicts the supposition that (2.2.37) does not hold, completing the proof.

Again, the obstacle to proving that (2.2.34) holds in general is the possibility of worse behavior, for example nondecaying oscillations or even growth of the error. We will see in the numerical experiments that (2.2.34) does accurately predict the behavior of the error for most of the conditions listed above.

It is worthwhile to consider the sensitivity of these results to the details of the model problem. The choice of a cylindrical scatterer is of no particular importance-one can study the solution for more general shapes using integral equation techniques. Of course, if there are trapped rays leading to slower decay rates for the transient solution, we may expect even slower decay of the error. Many authors use rectangular artificial boundaries and formulate their conditions in a way that is boundary dependent. This can have some effect on the behavior of the conditions. For example, condition (2.1.28) then leads to algebraic rather than exponential growth. However, the basic conclusion concerning the slow decay of the error for local in time, constant-coefficient conditions remains valid. More details can be found in Appendix B.

\section{CONSTRUCTION OF IMPROVED CONDITIONS}

In this section we construct variable-coefficient boundary conditions which have better long-time behavior than can be attained by constant-coefficient operators and, in addition, match a number of terms in the progressive wave (farfield) expansion. This guarantees their accuracy for short to moderate times. The subsequent numerical experiments clearly demonstrate their efficiency.

We assume a circular artificial boundary, $r=R$, and construct conditions for each Fourier mode. This will result in a spatially nonlocal operator, as is 
required if convergence to the correct steady state is to occur. We will also construct simplified approximations which allow small errors in the final state.

Let $v=\Re\left(\sum_{n=0}^{\infty} v_{n}(r, t) e^{i n \theta}\right)$. For $v_{n}$ we impose a boundary condition of the form

$$
\begin{aligned}
\mathscr{K}_{n} v_{n} & \equiv\left(\frac{\partial}{\partial t}+\delta_{n}(t)\right) \frac{\partial v_{n}}{\partial r}+\frac{\partial^{2} v_{n}}{\partial t^{2}}+\left(\frac{1}{2 R}+\delta_{n}(t)\right) \frac{\partial v_{n}}{\partial t}+\delta_{n}(t) G_{n}(t) v_{n} \\
& =0
\end{aligned}
$$

To determine $\delta_{n}$ and $G_{n}$, we consider separately the geometrical optics and long-time limits. The expansion (2.1.14) corresponds to the relationships

$$
\begin{gathered}
\frac{\partial^{2} u_{n}}{\partial t \partial r}+\frac{\partial^{2} u_{n}}{\partial t^{2}}+\frac{1}{2 R} \frac{\partial u_{n}}{\partial t}+\frac{4 n^{2}-1}{8 R^{2}} u_{n}=O\left(R^{-3} u_{n}\right), \\
\frac{\partial u_{n}}{\partial r}+\frac{\partial u_{n}}{\partial t}+\frac{1}{2 R} u_{n}=O\left(R^{-2} u_{n}\right) .
\end{gathered}
$$

Comparing these with (3.0.42) leads immediately to the condition

$$
\delta_{n}=\left(G_{n}-\frac{1}{2 R}\right)^{-1} \frac{4 n^{2}-1}{8 R^{2}},
$$

with the formal result

$$
\mathscr{K}_{n} u_{n}=O\left(\left(R^{-3}+\delta_{n} R^{-2}\right) u_{n}\right) .
$$

(This will become $O\left(R^{-3} u_{n}\right)$ as $\delta_{n}$ will scale like $R^{-1}$.)

To choose $G_{n}$, we consider the long-time near steady-state behavior. For (3.0.42) we have

$$
\frac{\partial v_{n}}{\partial r}+G_{n}(t) v_{n} \approx 0
$$

whereas (2.1.15) implies

$$
\begin{gathered}
\frac{\partial u_{n}}{\partial r}+\frac{n}{R} u_{n}=o\left(t^{-1}\right), \quad n \neq 0, \\
\frac{\partial u_{0}}{\partial r}+\int_{0}^{t} \mathscr{G}(t-p) u_{0}(R, p) d p=o\left(t^{-1}\right),
\end{gathered}
$$

where $\hat{\mathscr{G}}(s)=-\left(R\left(\ln \left(\frac{R s}{2}\right)+\gamma\right)\right)^{-1}+O\left(s^{2}\right)$. Equation (3.0.48) may be approximated using a constant $G_{n}$ :

$$
G_{n}=\frac{n}{R}, \quad \delta_{n}=\frac{(2 n+1)}{4 R}, \quad n \neq 0 .
$$

Equation (3.0.49), on the other hand, cannot be well approximated by (3.0.42) with constant $G_{0}$. (This is, of course, the content of Theorem 1.) However, as 
$u_{0}(R, t) \rightarrow u_{0}(R, \infty)$, we have $\hat{u}_{0}=\frac{1}{s} u_{0}(R, \infty)(1+o(1))$, so that

$$
\widehat{\mathscr{G} * u_{0}}=-\frac{u_{0}(R, \infty)}{R s\left(\ln \left(\frac{R s}{2}\right)+\gamma\right)} \cdot(1+o(1)) .
$$

Therefore, if we choose a decaying function $G_{0}(t)$ so that

$$
\hat{G}_{0}=-\frac{1}{R s\left(\ln \left(\frac{R s}{2}\right)+\gamma\right)} \cdot(1+o(1)),
$$

then $\widehat{G_{0} u_{0}}$ will agree with $\widehat{\mathscr{G} * u_{0}}$ to leading order for small $s$. We have already constructed a function with such behavor, $\mathscr{H}$. We avoid using $\mathscr{H}$ directly, as we have no fast means of evaluating it, but instead use its asymptotic expansion. That is, we take

$$
G_{0}(t) \sim-\frac{1}{R} \mathscr{H}\left(\frac{2 t}{R e^{\gamma}}\right)=\frac{1}{R \ln \left(\frac{2 t}{R}\right)}+O\left((\ln t)^{-3}\right) .
$$

(Note we have absorbed into one term the first two terms in the expansion of $\mathscr{H}$ in inverse powers of the log of its argument. This order of agreement determines the form of our final error estimate. A more accurate approximation would produce a more rapid asymptotic error decay.) Keeping in mind that $G_{0}-(2 R)^{-1} \neq 0$ must be enforced, we finally have

$$
\begin{gathered}
G_{0}(t)=\frac{1}{R \ln \zeta}, \quad \zeta=2 \frac{(t+D)}{R}, \quad D>\frac{R}{2} e^{2}, \\
\delta_{0}(t)=\frac{1}{4 R} \cdot \frac{1}{1-2(\ln \zeta)^{-1}} .
\end{gathered}
$$

(Here, $D$ is a free parameter. In the experiments it is taken to be $4.5 R$.)

Putting this all together we have our final form:

\section{Uniform asymptotic boundary condition.}

$$
\left(\frac{\partial}{\partial t}+\mathscr{D}(t)\right)\left(\frac{\partial v}{\partial r}+\frac{\partial v}{\partial t}+\frac{v}{2 R}\right)-\frac{1}{2 R^{2}} \frac{\partial^{2} v}{\partial \theta^{2}}-\frac{v}{8 R^{2}}=0
$$

where the nonlocal (in $\theta$ ) time-dependent operator $\mathscr{D}$ is defined by

$$
\mathscr{D} w(\theta, t)=\Re\left(\sum_{n=0}^{\infty} \delta_{n}(t) w_{n}(t) e^{i n \theta}\right),
$$

with

$$
w(\theta, t)=\Re\left(\sum_{n=0}^{\infty} w_{n}(t) e^{i n \theta}\right),
$$

and $\delta_{n}$ is defined by (3.0.50) or (3.0.55).

The main complication in using this condition, in comparison with other second-order operators, is the application of the nonlocal operator $\mathscr{D}$. Of course, this can be done using fast Fourier transforms, so that actual computational work is negligible. (See also Keller and Givoli [21] for the implementation of nonlocal conditions.) According to our analysis of the steady-state error for 
dissipative conditions (2.2.32), the accurate approximation of $b_{n}^{e}(0)$ is only important for small $n$. This may be used to simplify $\mathscr{D}$. A first (local) approximation is to replace $\mathscr{D}$ by $\delta_{0}$. The dominant part of the steady-state error will then correspond to $n=1$, have a maximum of $O\left(R^{-1}\right)$ and decay like $\frac{r}{R}$. The first two terms may be incorporated in an operator $\mathscr{D}_{1}$ :

$$
\mathscr{D}_{1} w(\theta, t)=\delta_{0}(t) \bar{w}(t)+\delta_{1}(w(\theta, t)-\bar{w}(t)), \quad \bar{w}(t)=\frac{1}{2 \pi} \int_{0}^{2 \pi} w(\theta, t) d \theta .
$$

Here we expect a maximum error of $O\left(R^{-2}\right)$ decaying like $\left(\frac{r}{R}\right)^{2}$. Of course, this approach can be generalized to an arbitrary finite number of modes. We emphasize, however, that there are no substantial savings in computational work for circular boundaries, though there may be in the general case.

In $\S 5$ we prove the well-posedness of the resulting initial-boundary value problem using energy estimates, and also prove that the transient error decays faster, $O\left((\ln t)^{-3}\right)$, than the lower bound established above for constant-coefficient operators. A similar construction for three-dimensional exterior problems is given in Appendix C.

\section{NUMERICAL EXPERIMENTS}

We have carried out a number of simple numerical experiments to test and illustrate the results derived in the preceding sections. These are necessary, as our long-time analysis is asymptotic and does not provide precise error bounds. We solved individually problems for various Fourier coefficients:

$$
\begin{aligned}
& \frac{\partial^{2} v_{n}}{\partial t^{2}}=\frac{1}{r} \frac{\partial}{\partial r}\left(r \frac{\partial v_{n}}{\partial r}\right)-\frac{n^{2}}{r^{2}} v_{n}, \quad 1<r<R, \\
& v_{n}(r, 0)=\frac{\partial v_{n}}{\partial t}(r, 0)=0, \quad v_{n}(1, t)=g(t) .
\end{aligned}
$$

In most of the experiments we have taken, for some $\omega$,

$$
g(t)=1-\frac{\cos 2 \pi \omega t}{1+t^{2}}
$$

All simulations were carried out to $t=500$, which was 50000 time steps. A standard second-order, centered finite difference discretization was employed with a uniform spatial mesh and $\frac{\Delta t}{\Delta r}=.5$. As we have a reasonably large number of points per wavelength (between 100 and 12 for the shortest waves), we are confident that the discretization errors are negligible in comparison with those due to the boundary conditions, particularly for long times, when the solution is very smooth. The boundary conditions used in the comparisons are (2.1.16), (2.1.19), (2.1.25), and (3.0.42). We also display a single simulation using (2.1.28) to illustrate the blow-up of the solution as $t \rightarrow \infty$. For reference we list in Appendix D the differencing used for each of these, all formulas being accurate to second order. For most of the results shown we have taken $R=8$. Note the we have carried out a large number of experiments, using other boundary conditions and varying $R$ and other parameters. What we present 


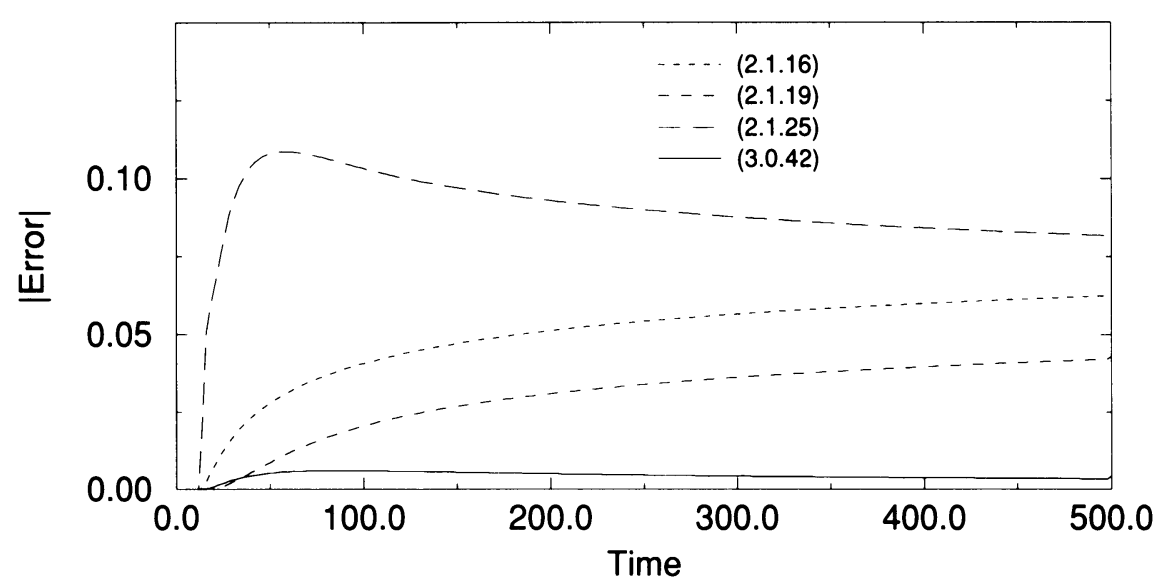

FiguRE 1. Errors for $n=0, \omega=1, R=8$

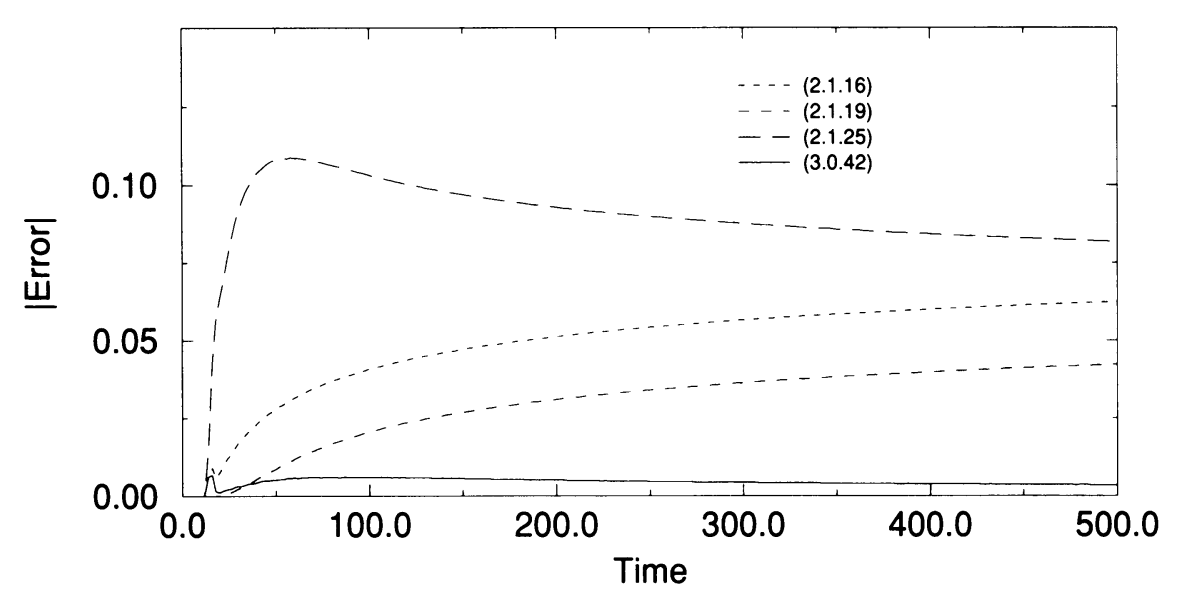

FIGURE 2. Errors for $n=0, \omega=4, R=8$

is both qualitatively and quantitatively representative of what was generally observed.

The advantage of solving the modal equations is the opportunity it provides for very long-time and (for comparison) very large-domain simulations at low computational cost. In particular, we have solved the difference equations with $R=252$ and the same uniform mesh ( 12551 points). The error due to the boundary condition is approximated by comparing these solutions every 20 time steps at the point $r=1.8$. We also checked the errors at a number of other stations, with essentially the same results. Of course, the errors we compute for each mode may be superposed to give the errors for a full simulation.

Figures 1-3 display the errors for $n=0$, which is the most difficult case. The behavior of the error for short times is clearly consistent with the degree to which the expansion (2.1.14) is matched by the boundary condition. That is, (2.1.19) is best followed by (3.0.42), (2.1.16), and (2.1.25). These differences are somewhat accentuated as the frequency, $\omega$, is made larger. The longtime error for all the constant-coefficient conditions is seen to be fairly large, 


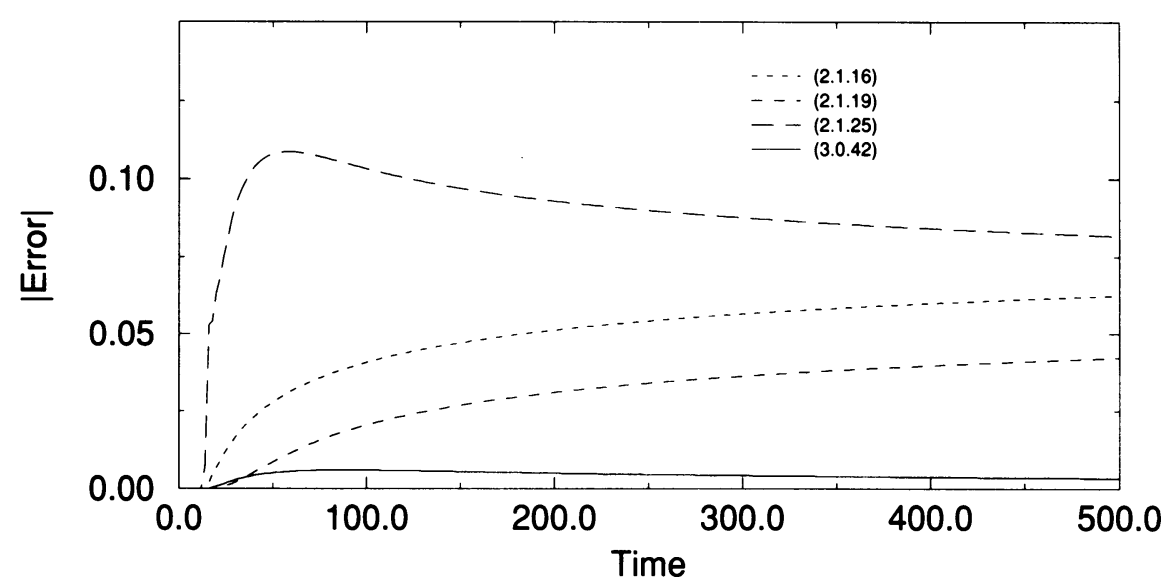

FIGURE 3. Errors for $n=0, \omega=\frac{1}{2}, R=8$

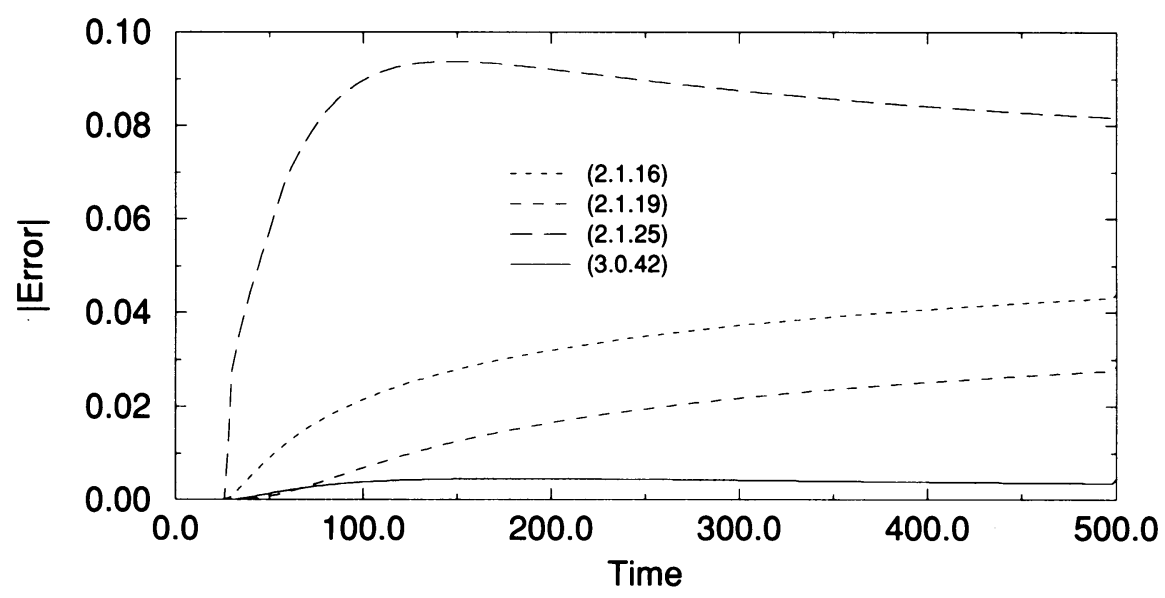

Figure 4. Errors for $n=0, \omega=1, R=16$

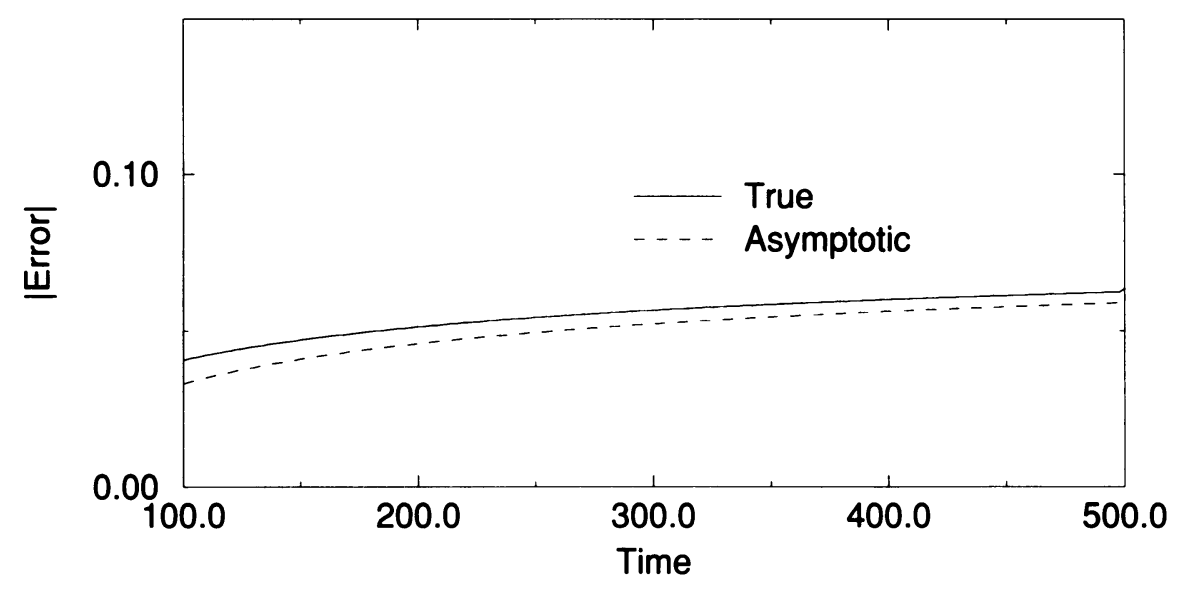

FIGURE 5. Asymptotic expansion vs. true error, condition (2.1.25) 


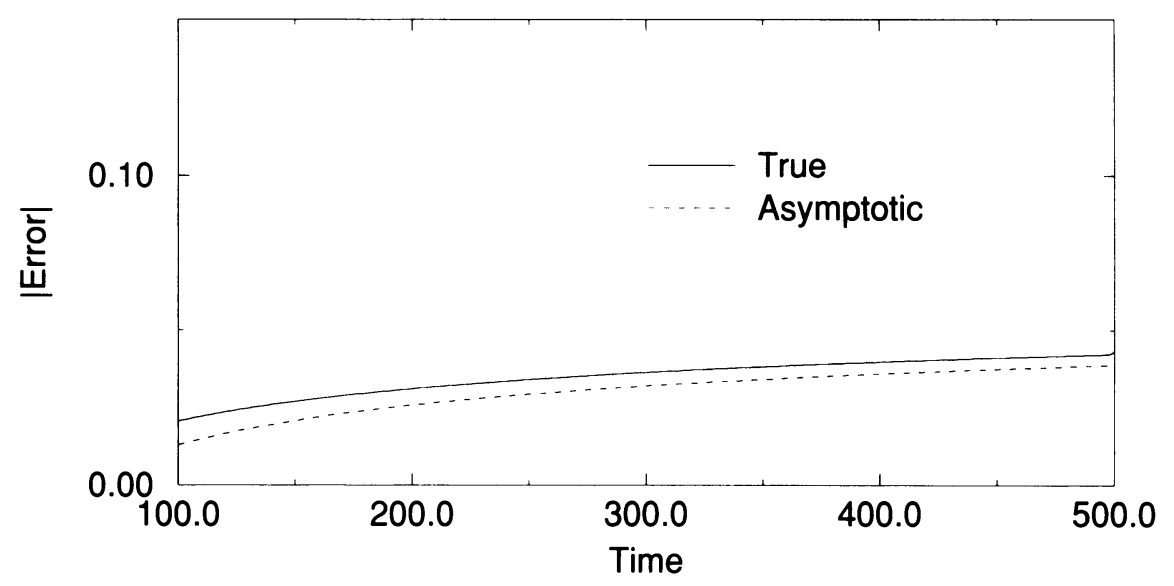

Figure 6. Asymptotic expansion vs. true error, condition (2.1.16)

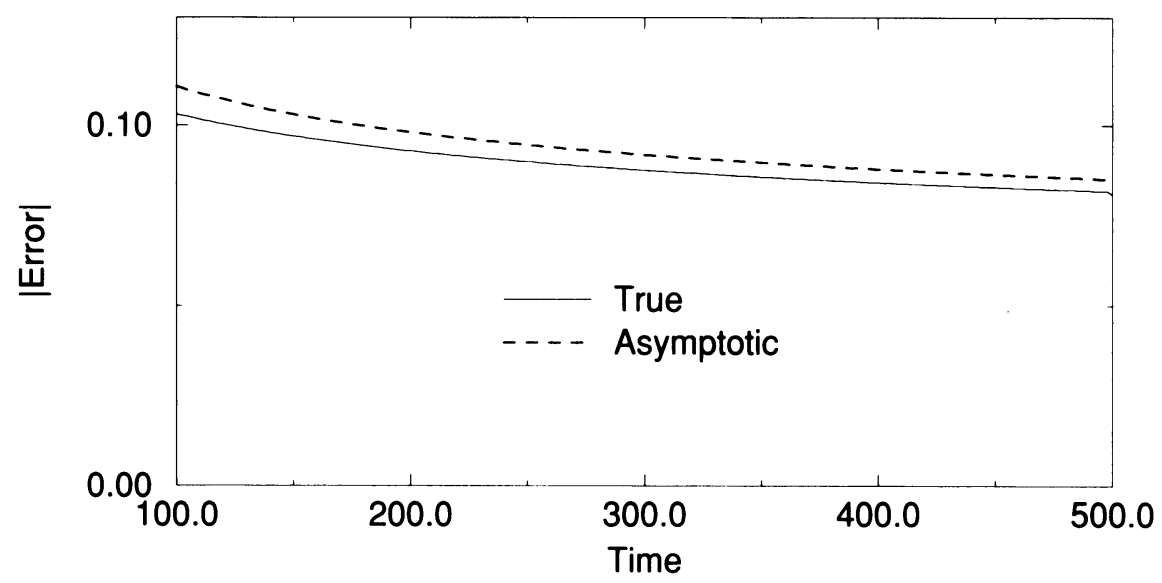

FIGURE 7. Asymptotic expansion vs. true error, condition (2.1.19)

ranging from $4 \%$ to $8 \%$ at $t=500$. (Note that the error from $(2.1 .25)$ is still largest, though its limiting value is 0 .) The error resulting from the corrected conditions, (3.0.42), is smaller than .4\%, an improvement of more than a factor of 10 . The insensitivity of these results to boundary location is illustrated by Figure 4, where we have doubled the domain radius.

A comparsion of the numerically computed error and the asymptotic expansion of the error (2.2.34) is presented in Figures 5-7. Plotted are the errors from Figure 1 versus

$$
\left|\left(\frac{b_{0}(0)}{1+b_{0}(0) \ln R}-\frac{1}{\ln 2 t}\right) \ln r\right| \text {. }
$$

Here, $b_{0}(0)$ is taken from the formulas in $\S 2, R=8, r=1.8$. The expression above is expected to be correct to $O\left((\ln t)^{-3}\right)$. We see that the candidate expansion does, in fact, correctly predict the behavior of the error for these particular boundary conditions. (Recall that in $\S 2$ we only prove that the error cannot decay faster.) 


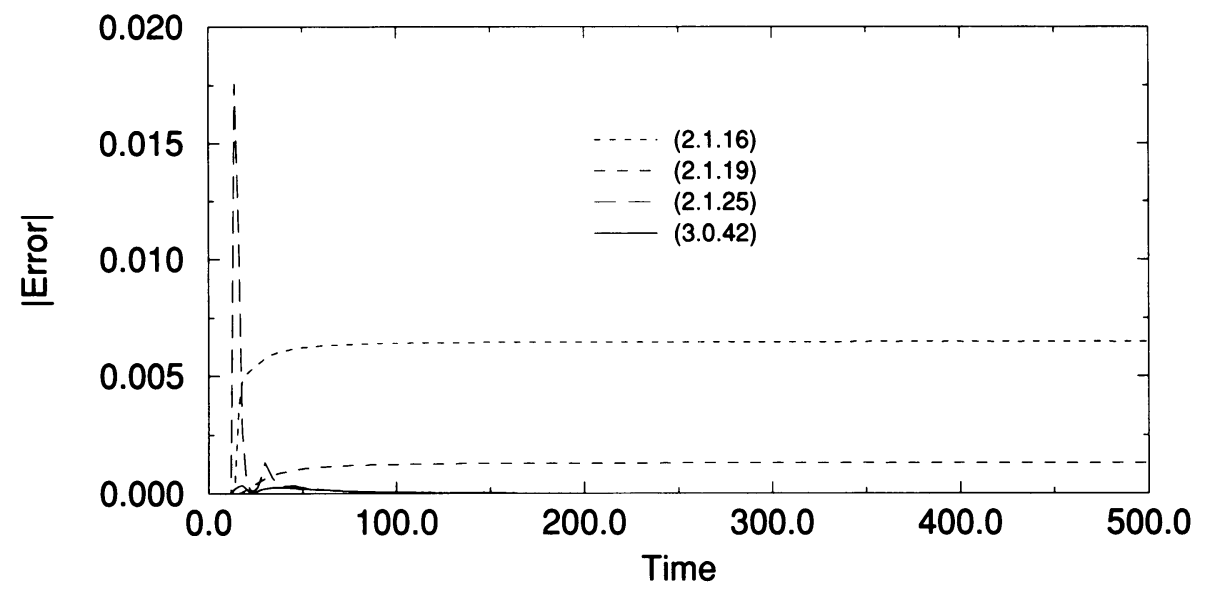

FIGURE 8. Errors for $n=1, \omega=1, R=8$

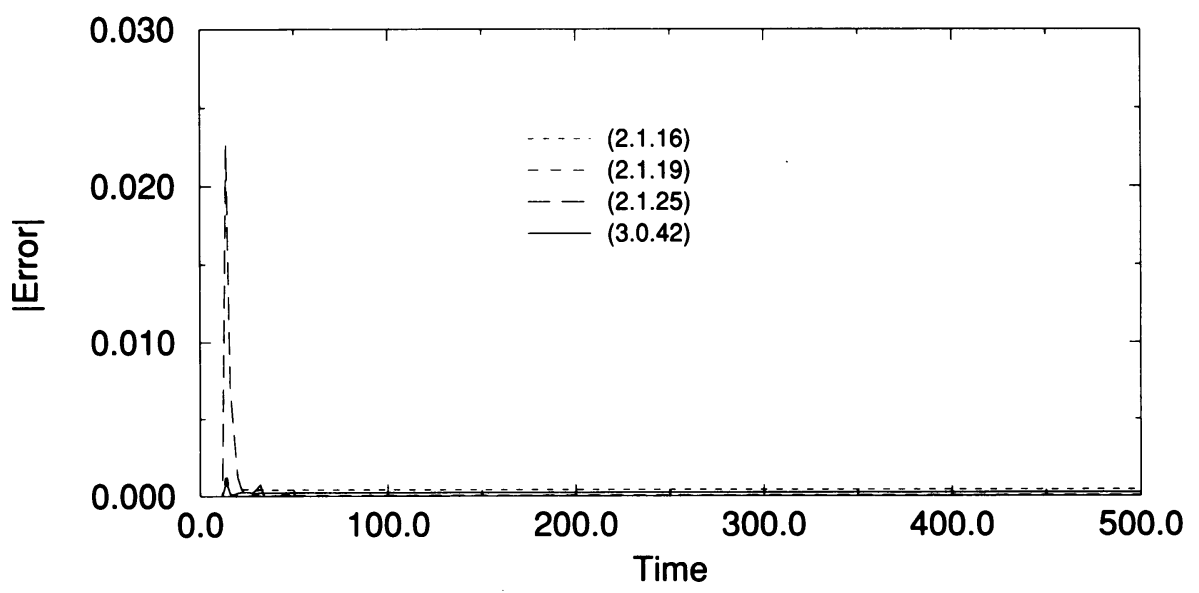

FIGURE 9. Errors for $n=2, \omega=1, R=8$

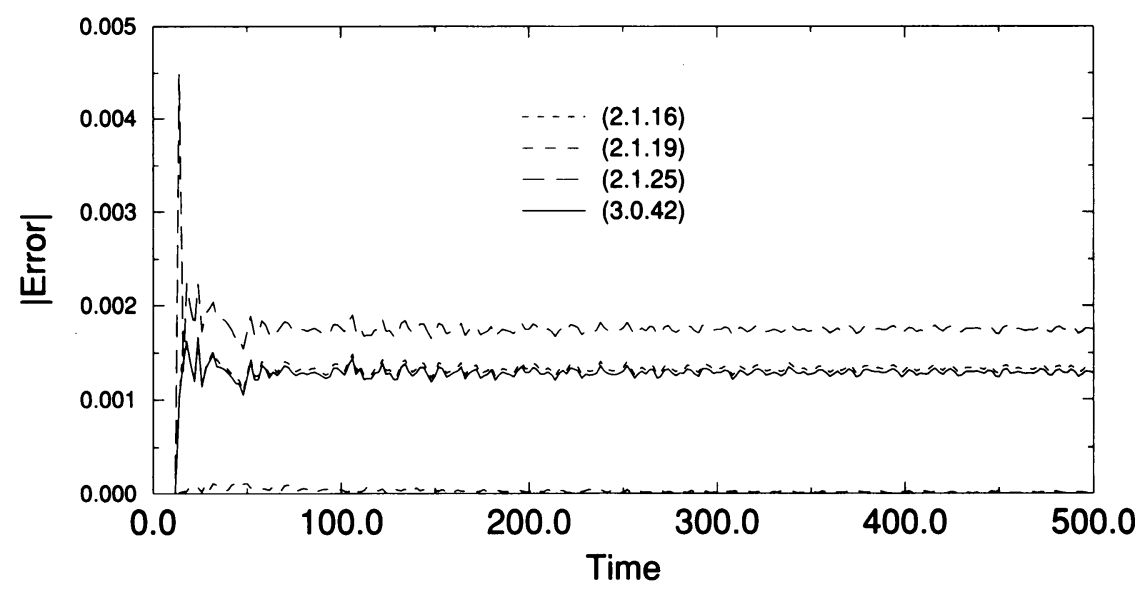

FIGURE 10. Errors for $n=0, g=\sin 2 \pi t, R=8$ 


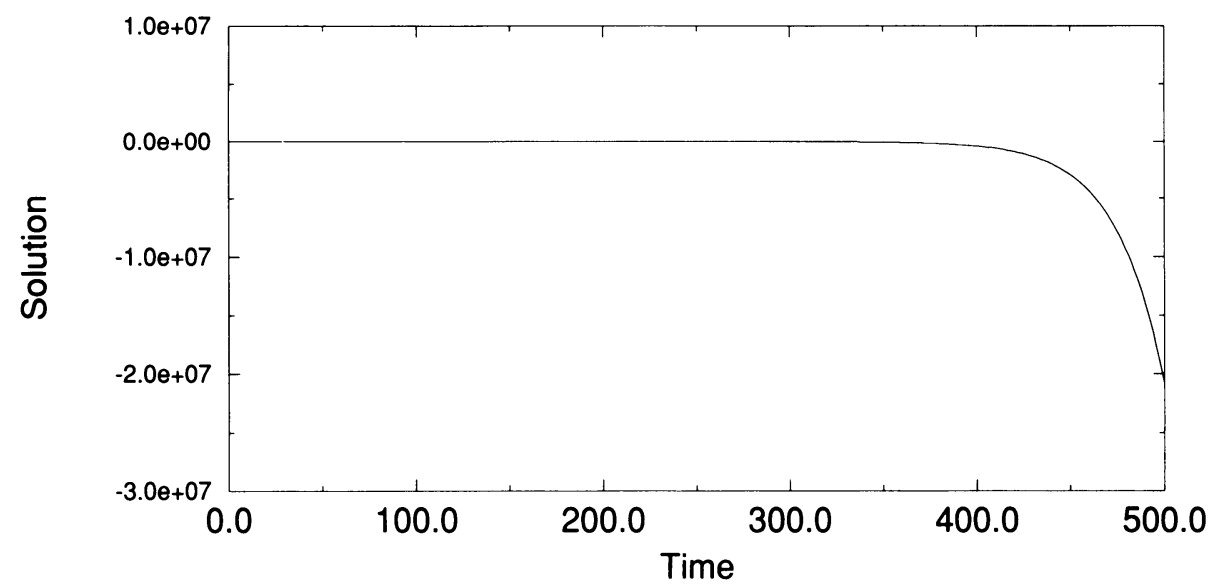

FIGURE 11. Solution at $r=1.8$ using (2.1.28), $n=0, \omega=1$, $R=8$

Errors for $n \neq 0$, in contrast, are much smaller and more rapidly decaying. This is illustrated in Figures 8-9, corresponding to $n=1,2$. Again, the overall performance of $(3.0 .42)$ is best.

We have also carried out simulations for a periodic forcing, $g=\sin 2 \pi t$. The results are shown in Figure 10. Here, the decay of the transients and the transient part of the error is more rapid. In this case we find that condition (2.1.19) is most accurate, corresponding to the nearly invisible curve at the bottom of the figure. It is followed by (3.0.42) and (2.1.16), which give nearly identical results. Again, we emphasize that the absolute size of the errors is small compared with the earlier experiments.

In our final example, Figure 11, we trace the solution at a particular point computed with the nondissipative condition, (2.1.28). We find, as expected, that the solution eventually grows exponentially in time, rendering the results meaningless.

\section{ENERGY ESTIMATES AND ERROR DECAY FOR VARIABLE-COEFFICIENT CONDITIONS}

In this section we derive energy estimates for our proposed boundary conditions. First of all, these imply the well-posedness of the resulting initialboundary value problem. Secondly, we use them to prove that our condition is dissipative, that is, that the solution approaches the correct steady state, and that the long-time behavior of the error is better than can be obtained with constant-coefficient operators (see Theorem 1). The proof is somewhat complex, relying on different techniques for the variable- and constant-coefficient parts of the boundary condition. To facilitate the decomposition of the error into $\theta$-independent and $\theta$-dependent pieces, we only consider the case of a circular scatterer.

We seek to approximate the solution, $u$, to

$$
\frac{\partial^{2} u}{\partial t^{2}}=\nabla^{2} u+f
$$


exterior to a disk, $\Omega$, of radius 1 . We also have initial and boundary conditions (using the usual polar coordinates $r$ and $\theta$ with origin at the disk center):

$$
u=g, \quad r=1, \quad u(\vec{x}, 0)=w_{0}(\vec{x}), \quad \frac{\partial u}{\partial t}(\vec{x}, 0)=w_{1}(\vec{x}) .
$$

We further suppose that $f, w_{i}$ have compact support, that $f, g$ approach limits as $t \rightarrow \infty$ sufficiently fast and that all the data is smooth.

Introducing a circular artificial boundary at $r=R$ such that the support of the data is contained within the truncated domain, we approximate $u$ by $v$, where $v$ satisfies the wave equation with the same data as $u$ and, in addition, (3.0.56) at $r=R$. The error, $e=u-v$, then satisfies

$$
\begin{gathered}
\frac{\partial^{2} e}{\partial t^{2}}=\nabla^{2} e, \quad e(\vec{x}, 0)=\frac{\partial e}{\partial t}(\vec{x}, 0)=0, \quad \vec{x} \in \Upsilon, \\
e=0, \quad r=1, \quad B e=B u \equiv \Psi(\theta, t), \quad r=R,
\end{gathered}
$$

where we denote by $B$ the operator in (3.0.56) and $\Upsilon$ is the annulus given by $1<r<R$. We decompose $e$ into two orthogonal parts, both of which satisfy the homogeneous wave equation with zero data away from the artificial boundary:

$$
e(r, \theta, t)=e_{0}(r, t)+\tilde{e}(r, \theta, t),
$$

where $e_{0}(r, t)=(1 / 2 \pi) \int_{0}^{2 \pi} e(r, \theta, t) d \theta$. The boundary conditions satisfied by $e_{0}$ and $\tilde{e}$ are

$$
\left(\frac{\partial}{\partial t}+\delta_{0}(t)\right)\left(\frac{\partial e_{0}}{\partial r}+\frac{\partial e_{0}}{\partial t}+\frac{e_{0}}{2 R}\right)-\frac{e_{0}}{8 R^{2}}=\Psi_{0}(t) \equiv \frac{1}{2 \pi} \int_{0}^{2 \pi} \Psi(\theta, t) d \theta,
$$

$$
\begin{aligned}
\left(\frac{\partial}{\partial t}\right. & +\overline{\mathscr{D}})\left(\frac{\partial \tilde{e}}{\partial r}+\frac{\partial \tilde{e}}{\partial t}+\frac{\tilde{e}}{2 R}\right)-\frac{1}{2 R^{2}} \frac{\partial^{2} \tilde{e}}{\partial \theta^{2}}-\frac{\tilde{e}}{8 R^{2}} \\
& =\tilde{\Psi}(\theta, t) \\
& \equiv \Psi(\theta, t)-\Psi_{0}(\theta, t) .
\end{aligned}
$$

Here, the constant-coefficient nonlocal operator $\bar{D}$ is given by the sum in (3.0.57) excluding the $n=0$ term.

The estimates for $e_{0}$ and $\tilde{\boldsymbol{e}}$ will be made separately, using quite different arguments. We note that both energy estimates could be made with a general star-shaped scatterer, but we would be unable to make the simple decomposition of the error into its Fourier modes. We expect that $e_{0}$ will represent the dominant error for long times, and so we will consider it first. 
5.1. Estimates for $e_{0}$. We employ a one-parameter family of energies adapted from Muravei [26]:

$$
\begin{aligned}
\mathscr{E}(t ; K) \equiv \frac{1}{2} \int_{1}^{R}\left[\left(\bar{t}^{2}+r^{2}\right)\left(\left(\frac{\partial e_{0}}{\partial t}\right)^{2}+\left(\frac{\partial e_{0}}{\partial r}\right)^{2}\right)\right. \\
\left.+4 \bar{t} r \frac{\partial e_{0}}{\partial t} \frac{\partial e_{0}}{\partial r}+2 \bar{t} e_{0} \frac{\partial e_{0}}{\partial t}-e_{0}^{2}\right] r d r
\end{aligned}
$$

where $\bar{t}=t+K$ and $K$ is eventually to be chosen sufficiently large. Differentiating $\mathscr{E}$ with respect to $t$, using the equation satisfied by $e_{0}$, integrating by parts, and applying the boundary condition at $\partial \Omega$, we obtain the fundamental identity

$$
\begin{aligned}
\dot{\mathscr{E}} & =R\left[\left(\bar{t}^{2}+R^{2}\right) \frac{\partial e_{0}}{\partial t} \frac{\partial e_{0}}{\partial r}+R \bar{t}\left(\left(\frac{\partial e_{0}}{\partial t}\right)^{2}+\left(\frac{\partial e_{0}}{\partial r}\right)^{2}\right)+\bar{t} e_{0} \frac{\partial e_{0}}{\partial r}\right]_{r=R}-\left.\bar{t}\left(\frac{\partial e_{0}}{\partial r}\right)^{2}\right|_{r=1} \\
& \left.=R\left[\hat{t}^{2} \frac{\partial e_{0}}{\partial t} \frac{\partial e_{0}}{\partial r}+R \bar{t}\left(\frac{\partial e_{0}}{\partial t}+\frac{\partial e_{0}}{\partial r}\right)\right)^{2}+\bar{t} e_{0} \frac{\partial e_{0}}{\partial r}\right]_{r=R}-\left.\bar{t}\left(\frac{\partial e_{0}}{\partial r}\right)^{2}\right|_{r=1} .
\end{aligned}
$$

We have introduced $\hat{t}=\bar{t}-R$.

We must now use our boundary condition at $r=R$ to estimate the energy derivative. As a preliminary step, we first rewrite the boundary condition in integral form by inverting the differential operator $\left(\frac{\partial}{\partial t}+\delta_{0}(t)\right)$ :

$$
\frac{\partial e_{0}}{\partial t}+\frac{\partial e_{0}}{\partial r}+\frac{1}{2 R} e_{0}-\frac{1}{8 R^{2}} \int_{0}^{t} e^{-\int_{\tau}^{t} \delta_{0}(\eta) d \eta} e_{0}(R, \tau) d \tau=\bar{\Psi}_{0}(t)
$$

and performing an integration by parts:

$$
\frac{\partial e_{0}}{\partial t}+\frac{\partial e_{0}}{\partial r}+q(t) e_{0}+\frac{1}{8 R^{2}} \int_{0}^{t} Z(t, \tau) \frac{\partial e_{0}}{\partial \tau}(R, \tau) d \tau=\bar{\Psi}_{0}(t) .
$$

Here we have introduced

$$
Z(t, \tau)=\int_{0}^{\tau} e^{-\int_{s}^{t} \delta_{0}(\eta) d \eta} d s, \quad q(t)=\frac{1}{2 R}\left(1-\frac{1}{4 R} Z(t, t)\right),
$$

as well as the modified data,

$$
\bar{\Psi}_{0}(t)=\int_{0}^{t} e^{-\int_{s}^{t} \delta_{0}(\eta) d \eta} \Psi_{0}(s) d s .
$$

It is convenient to state and prove here certain facts about $q$ which will be useful later on:

Lemma 1. There holds

$$
q(t) \geq 0, \quad q^{\prime}(t) \leq 0, \quad q=O(1 /(R \ln (t / R))), \quad t \rightarrow \infty,
$$


uniformly in $R$, and, for any $\epsilon>0, K=\hat{K}(\epsilon) R^{2}$ can be chosen sufficiently large such that

$$
(t+K-R) q^{\prime}(t)+2 q(t)+\frac{1}{t+K-R} \leq \frac{\epsilon}{R \ln R}
$$

Proof. We recall that $\delta_{0}(t)$ is given by (3.0.55) and satisfies

$$
\delta_{0}(t) \geq \frac{1}{4 R}, \quad \delta_{0}^{\prime}(t) \leq 0, \quad \delta_{0}(t)=\frac{1}{4 R}(1+O(1 / \ln (t / R))), t \rightarrow \infty .
$$

This implies

$$
Z(t, t) \leq \int_{0}^{t} e^{-\delta_{0}(t)(t-s)} d s=\frac{1}{\delta_{0}(t)}\left(1-e^{-\delta_{0}(t) t}\right) \leq 4 R
$$

which immediately yields $q \geq 0$. Differentiating $q$ and using (5.1.80), we obtain

$$
q^{\prime}=-\frac{1}{8 R^{2}} \frac{d}{d t} Z(t, t)=-\frac{1}{8 R^{2}}\left(1-\delta_{0}(t) Z(t, t)\right) \leq 0
$$

Now, integrating by parts, we get

$$
Z(t, t)=\frac{1}{\delta_{0}(t)}+\frac{\delta_{0}^{\prime}(t)}{\delta_{0}^{3}(t)}-\int_{0}^{t}\left(\frac{\delta_{0}^{\prime}(s)}{\delta_{0}^{3}(s)}\right)^{\prime} e^{-\int_{s}^{t} \delta_{0}(\eta) d \eta} d s+O\left(e^{-\int_{0}^{t} \delta_{0}(\eta) d \eta}\right)
$$

The first term on the right-hand side is $4 R(1+O(1 / \ln (t / R)))$ as $t \rightarrow \infty$, and the second is $O\left(R /\left((t / R) \ln ^{2}(t / R)\right)\right)$. Putting this into the formula for $q$ yields $q=O(1 /(R \ln (t / R)))$.

Finally, we consider the terms on the left-hand side of (5.1.78). Using the expansion for $Z$ and introducing $\tilde{t}=(t+D) / R$ and $\bar{K}=O(K / R), R \rightarrow \infty$, we obtain, for some positive constants $\gamma_{1}, \gamma_{2}$ independent of $R$ and $K$,

$$
\begin{gathered}
2 q \leq \frac{\gamma_{1}}{R \ln \tilde{t}}, \\
(t+K-R) q^{\prime} \leq-\gamma_{2}(1+(\bar{K} / \tilde{t})) \frac{1}{R \ln ^{2} \tilde{t}} .
\end{gathered}
$$

(Here we use the fact that $D=O(R)$.) Combining these and taking $\bar{K}=$ $O(R)$ sufficiently large, we see that $(t+K-R) q^{\prime}+2 q$ is negative until $\tilde{t}=$ $O(\bar{K} / \ln \bar{K})$. Its maximum, then, is $O(1 /(R \ln \bar{K}))=O(1 /(R \ln R))$, and can be made arbitrarily small by choosing $K /\left(R^{2}\right)$ sufficiently large. Since the maximum of the remaining term behaves like $1 / K$, the estimate holds. This completes the proof of Lemma 1.

Solving for $\frac{\partial e_{0}}{\partial r}$ in $(5.1 .73,5.1 .74)$, substituting in (5.1.72), and integrating in time from 0 to $T$, we obtain 


$$
\begin{aligned}
\mathscr{E}(T) & +\left.\int_{0}^{T} \bar{t}\left(\frac{\partial e_{0}}{\partial r}\right)^{2}\right|_{r=1} d t+\left.\int_{0}^{T} \hat{t}^{2} R\left(\frac{\partial e_{0}}{\partial t}\right)^{2}\right|_{r=R} d t \\
= & -\left.\frac{1}{2} \int_{0}^{T}\left(\hat{t}^{2} q(t)+\bar{t}\right) R\left(\frac{\partial\left(e_{0}^{2}\right)}{\partial t}\right)\right|_{r=R} d t-\left.\frac{1}{2} \int_{0}^{T} \bar{t}\left(e_{0}\right)^{2}\right|_{r=R} d t \\
& +\left.\int_{0}^{T} \hat{t}^{2} R\left(\frac{\partial e_{0}}{\partial t} \cdot \bar{\Psi}_{0}\right)\right|_{r=R} d t \\
& -\frac{1}{8 R} \int_{0}^{T} \hat{t}^{2}\left(\frac{\partial e_{0}}{\partial t}(R, t) \cdot\left(\int_{0}^{t} Z(t, \tau) \frac{\partial e_{0}}{\partial \tau}(R, \tau) d \tau\right)\right) d t \\
& +R^{2} \int_{0}^{T} \bar{t}\left(\bar{\Psi}_{0}(t)-\frac{1}{2 R} e_{0}(R, t)+\frac{1}{8 R^{2}} \int_{0}^{t} e^{-\int_{s}^{t} \delta_{0}(\eta) d \eta} e_{0}(R, s) d s\right)^{2} d t \\
& +\frac{1}{8 R} \int_{0}^{T} \bar{t}\left(e_{0}(R, T) \cdot\left(\int_{0}^{t} e^{-\int_{s}^{t} \delta_{0}(\eta) d \eta} e_{0}(R, s) d s\right)\right) d t \\
& +\int_{0}^{T} \bar{t} e_{0}(R, t) \cdot \bar{\Psi}_{0}(t) d t .
\end{aligned}
$$

Our strategy from this point on is simple. We will show that the terms on the right-hand side of $(5.1 .85)$ involving $\frac{\partial e_{0}}{\partial t}$ are, in aggregate, negative, and we will use $\mathscr{E}$ to estimate the terms involving $e_{0}$. Throughout, $\epsilon$ and $G$ will stand for constants independent of $t, R, e_{0}$ and $\bar{\Psi}_{0}$ such that $\epsilon$ can be made arbitrarily small (typically at the cost of making $K$ and $G$ large). The basic estimates are presented in a sequence of lemmas.

Lemma 2. We have

$$
\begin{aligned}
& -\frac{1}{2} \int_{0}^{T}\left(\hat{t}^{2} q(t)+\bar{t}\right) R\left(\frac{\partial\left(e_{0}^{2}\right)}{\partial t}(R, t)\right) d t \\
& \quad \leq-\frac{1}{2}\left(\hat{T}^{2} q(T)+T\right) R e_{0}^{2}(R, T)+\frac{\epsilon}{R \ln R} \int_{0}^{T} \hat{t} R e_{0}^{2}(R, t) d t
\end{aligned}
$$

Proof. Simply integrate by parts and apply the second part of Lemma 1, choosing $K$ sufficiently large.

Lemma 3. We have

$$
\int_{0}^{T} \hat{t}^{2} R\left(\frac{\partial e_{0}}{\partial t}(R, t) \cdot\left(\int_{0}^{t} Z(t, \tau) \frac{\partial e_{0}}{\partial \tau}(R, \tau) d \tau\right)\right) d t \geq 0
$$

Proof. Let

$$
w(t)=\hat{t}^{2} \frac{\partial e_{0}}{\partial t}(R, t), \quad \tilde{Z}(t, \tau)=\frac{1}{\hat{\tau}^{2}} Z(t, \tau)
$$

It is sufficient to show that

$$
\int_{0}^{T} w(t) \int_{0}^{t} \tilde{Z}(t, \tau) w(\tau) d \tau d t \geq 0
$$


for all functions $w$. By a result from the theory of Volterra equations (see Gripenberg, Londen, and Staffans [11, §20.2, Theorem 2.2]), it is sufficient to show that, for $t \geq \tau \geq 0$,

$$
\tilde{Z} \geq 0, \quad \frac{\partial \tilde{Z}}{\partial t} \leq 0, \quad \frac{\partial \tilde{Z}}{\partial \tau} \geq 0, \quad \frac{\partial^{2} \tilde{Z}}{\partial t \partial \tau} \leq 0 .
$$

Recall that $\tilde{Z}=(\tau+K-R)^{-2} \int_{0}^{\tau} e^{-\int_{s}^{t} \delta_{0}(\eta) d \eta} d s$. Clearly $\tilde{Z} \geq 0$ for $K>R$. Differentiating and recalling that $\delta_{0}>0$, we obtain

$$
\begin{gathered}
\frac{\partial \tilde{Z}}{\partial t}=-\delta_{0}(t) \tilde{Z} \leq 0, \\
\frac{\partial^{2} \tilde{Z}}{\partial t \partial \tau}=-\delta_{0}(t) \frac{\partial \tilde{Z}}{\partial \tau} .
\end{gathered}
$$

We need only show, then, that the $\tau$-derivative of $\tilde{Z}$ is nonnegative. We have, using (5.1.80)

$$
\begin{aligned}
\frac{\partial \tilde{Z}}{\partial \tau} & =\hat{\tau}^{-3} e^{-\int_{\tau}^{t} \delta_{0}(\eta) d \eta}(\tau+K-R-2 Z(\tau, \tau)) \\
& \geq \hat{\tau}^{-3} e^{-\int_{\tau}^{t} \delta_{0}(\eta) d \eta}(\tau+K-9 R)
\end{aligned}
$$

which will be nonnegative for $K \geq 9 R$, completing the proof.

Lemma 4. We have

$$
\begin{aligned}
\int_{0}^{T} \hat{t}^{2} R & \frac{\partial e_{0}}{\partial t}(R, t) \cdot \bar{\Psi}_{0}(t) d t \\
\leq & \frac{\epsilon}{\ln R}\left(\hat{T}^{2} e_{0}^{2}(R, T)+\int_{0}^{T} \hat{t} e_{0}^{2}(R, t) d t\right) \\
& +G R^{2} \ln R\left(\hat{T}^{2} \bar{\Psi}_{0}^{2}(T)+\int_{0}^{T} \hat{t} \bar{\Psi}_{0}^{2}(t) d t+\int_{0}^{T} \hat{t}^{3}\left(\frac{\partial \bar{\Psi}_{0}}{\partial t}(t)\right)^{2} d t\right) .
\end{aligned}
$$

Proof. Integration by parts in $t$ yields that the left-hand side of the expression above is equal to

$$
\begin{aligned}
R\left(\hat{T}^{2} e_{0}(R, T) \bar{\Psi}_{0}(T)\right. & -2 \int_{0}^{T} \hat{t} e_{0}(R, t) \bar{\Psi}_{0}(t) d t \\
& \left.-\int_{0}^{T} \hat{t}^{2} e_{0}(R, t) \frac{\partial \bar{\Psi}_{0}}{\partial t}(t) d t\right)
\end{aligned}
$$

The final result follows from the elementary product inequality

$$
a b \leq \epsilon a^{2}+\frac{1}{4 \epsilon} b^{2}
$$


Lemma 5. We have

$$
\begin{aligned}
\int_{0}^{T} & \bar{t} R\left(\int_{0}^{t} e^{-\int_{s}^{t} \delta_{0}(\eta) d \eta} e_{0}(R, s) d s\right)^{2} d t \\
& \leq 16 R^{3}\left(1+\frac{\epsilon}{\ln R}\right) \int_{0}^{T} \bar{t} e_{0}^{2}(R, t) d t .
\end{aligned}
$$

Proof. We have, for $s \leq t$,

$$
(t+K) \leq(s+K) \frac{(t-s+K)}{K}, \quad e^{-\int_{s}^{t} \delta_{0}(\eta) d \eta} \leq e^{-\frac{(t-s)}{4 R}}
$$

Therefore, the quantity to be estimated is bounded above by

$$
\frac{R}{K} \int_{0}^{T}\left(\Xi *(s+K)^{\frac{1}{2}}\left|e_{0}(R, s)\right|\right)^{2} d t
$$

where $\Xi(s)=(s+K)^{\frac{1}{2}} e^{-\frac{s}{4 R}}$ and $*$ denotes convolution in the time variable. By standard estimates for convolutions [11, $§ 2.2$, Theorem 2.2] this is bounded above by

$$
\frac{R}{K}\left(\int_{0}^{T} \Xi(s) d s\right)^{2} \int_{0}^{T} \bar{t} e_{0}^{2}(R, t) d t
$$

Integrating by parts gives

$$
\begin{aligned}
\int_{0}^{T}(s+K)^{\frac{1}{2}} e^{-\frac{s}{4 R}} d s= & -\left.4 R e^{-\frac{s}{4 R}}\left((s+K)^{\frac{1}{2}}+2 R(s+K)^{-\frac{1}{2}}\right)\right|_{0} ^{T} \\
& -4 R^{2} \int_{0}^{T}(s+K)^{-\frac{3}{2}} e^{-\frac{s}{4 R}} d s \\
\leq & 4 R K^{\frac{1}{2}}\left(1+\frac{2 R}{K}\right)
\end{aligned}
$$

Substituting this into the previous expression and choosing $K=O(R \ln R)$ sufficiently large yields the desired result.

Lemma 6. We have

$$
\begin{aligned}
\int_{0}^{T} \bar{t} & R e_{0}(R, t) \cdot \bar{\Psi}_{0}(t) d t+\frac{1}{8 R} \int_{0}^{T} \bar{t} e_{0}(R, t) \cdot\left(\int_{0}^{t} e^{-\int_{s}^{t} \delta_{0}(\eta) d \eta} e_{0}(R, s) d s\right) d t \\
& +R^{2} \int_{0}^{T} \bar{t}\left(\bar{\Psi}_{0}(t)-\frac{1}{2 R} e_{0}(R, t)+\frac{1}{8 R^{2}} \int_{0}^{t} e^{-\int_{s}^{t} \delta_{0}(\eta) d \eta} e_{0}(R, s) d s\right)^{2} d t \\
\leq & \left(\frac{1}{2}+\frac{\epsilon}{\ln R}\right) \int_{0}^{T} \bar{t} e_{0}^{2}(R, t) d t+G R^{2} \ln R \int_{0}^{T} \bar{t} \bar{\Psi}_{0}^{2}(t) d t
\end{aligned}
$$


Proof. The terms involving $\bar{\Psi}_{0}$ may all be estimated using the product inequality and Lemma 5. Expanding the remaining terms yields

$$
\frac{1}{4} \int_{0}^{T} \bar{t} e_{0}^{2}(R, t) d t+\frac{1}{64 R^{2}} \int_{0}^{T} \bar{t}\left(\int_{0}^{t} e^{-\int_{s}^{t} \delta_{0}(\eta) d \eta} e_{0}(R, s) d s\right)^{2} d t
$$

The final estimate is then a direct consequence of Lemma 5.

Putting all these inequalities into (5.1.85), we finally obtain

Theorem 2. For any $\epsilon>0$ and $K=O\left(R^{2}\right)$ sufficiently large, there exists a constant $G$ independent of $T, R$, and $\bar{\Psi}_{0}$ such that

$$
\begin{aligned}
\mathscr{E}(T)+ & \int_{0}^{T} \bar{t}\left(\frac{\partial e_{0}}{\partial r}(1, t)\right)^{2} d t+\int_{0}^{T} \bar{t}^{2} R\left(\frac{\partial e_{0}}{\partial t}(R, t)\right)^{2} d t \\
\leq & \frac{\epsilon}{\ln R}\left(\bar{T}^{2} e_{0}^{2}(R, T)+\int_{0}^{T} \bar{t} e_{0}^{2}(R, t) d t\right) \\
& +G R^{2} \ln R\left(\bar{T}^{2} \bar{\Psi}_{0}^{2}(T)+\int_{0}^{T} \bar{t} \bar{\Psi}_{0}^{2}(t) d t+\int_{0}^{T} \bar{t}^{3}\left(\frac{\partial \bar{\Psi}_{0}}{\partial t}(t)\right)^{2} d t\right) .
\end{aligned}
$$

We now use this energy inequality to finally estimate $e_{0}$ in terms of $\bar{\Psi}_{0}$. For economy of expression we introduce $\mathscr{G}\left(T ; \bar{\Psi}_{0}\right)$ to denote the terms in parentheses on the last line of (5.1.103). The first step is to prove a Poincarétype inequality relating $e_{0}$ at $R$ to $\mathscr{E}$.

Lemma 7. For $K=O(R \ln R)$ sufficiently large, there exists a constant $L$ independent of $R, T$, and $e_{0}$ such that

$$
\bar{t}^{2} e_{0}^{2}(r, t) \leq L \ln r \cdot \mathscr{E}(t ; K), \quad 1 \leq r \leq R .
$$

Proof. We begin by estimating $e_{0}$ in terms of $\int r\left(\frac{\partial e_{0}}{\partial r}\right)^{2}$. We have

$$
e_{0}^{2}(R, t)=\left[\int_{1}^{R} \frac{\partial e_{0}}{\partial r}(r, t) d r\right]^{2} \leq \ln R \int_{1}^{R}\left(\frac{\partial e_{0}}{\partial r}(r, t)\right)^{2} r d r
$$

By the same techniques we find that

$$
\int_{1}^{R} e_{0}^{2} r d r \leq \frac{R^{2} \ln R}{2} \int_{1}^{R}\left(\frac{\partial e_{0}}{\partial r}\right)^{2} r d r .
$$

Combining this with the elementary inequalities

$$
\begin{aligned}
& \left|4 r \bar{t} \frac{\partial e_{0}}{\partial r} \frac{\partial e_{0}}{\partial t}\right| \leq 2 r \bar{t}\left(\left(\frac{\partial e_{0}}{\partial r}\right)^{2}+\left(\frac{\partial e_{0}}{\partial t}\right)^{2}\right), \\
& \left|2 \bar{t} e_{0} \frac{\partial e_{0}}{\partial t}\right| \leq 4 e_{0}^{2}+\frac{\bar{t}^{2}}{4}\left(\frac{\partial e_{0}}{\partial t}\right)^{2}
\end{aligned}
$$

yields 
(5.1.108)

$$
\mathscr{E} \geq \frac{1}{2} \int_{1}^{R}\left[\frac{3}{4}(\bar{t}-r)^{2}\left(\left(\frac{\partial e_{0}}{\partial t}\right)^{2}+\left(\frac{\partial e_{0}}{\partial r}\right)^{2}\right)-\frac{5}{2} R^{2} \ln R\left(\frac{\partial e_{0}}{\partial r}\right)^{2}\right] r d r .
$$

Choosing $K=O(R \ln R)$ sufficiently large keeps $(\bar{t}-r)^{2}>M R^{2} \ln R$ for any $M$ so that for some constant $L$ independent of $R$ and $T$ we have

$$
\int_{1}^{R} \bar{t}^{2}\left(\frac{\partial e_{0}}{\partial r}\right)^{2} r d r \leq L \cdot \mathscr{E}(t ; K) .
$$

Substituting this into (5.1.105) yields the desired result for $r=R$. The extension to $r<R$ follows directly since the integrals used to bound the left-hand side all had nonnegative integrands.

Remark. The calculation used in Lemma 7 shows that, for $K$ sufficiently large, the quantities $\int_{1}^{R} r\left(\frac{\partial e_{0}}{\partial r}(r, t)\right)^{2} d r, \int_{1}^{R} r\left(\frac{\partial e_{0}}{\partial t}(r, t)\right)^{2} d r$ and $\left|e_{0}(r, t)\right|$ are all bounded by $\mathscr{E}(T, K) \cdot T^{-2}$.

We are now able to estimate $\mathscr{E}$ directly in terms of $\Psi_{0}$ :

Theorem 3. For any $\epsilon>0$ we can choose $K=\hat{K}(\epsilon) R^{2}$ sufficiently large such that

$$
\mathscr{E}(T ; K) \leq G(\epsilon) R \ln R \cdot T^{\epsilon} \int_{0}^{T} \bar{t}^{-\epsilon} \frac{d \mathscr{G}}{d t}\left(t ; \bar{\Psi}_{0}\right) d t .
$$

Proof. Making $\epsilon$ in (5.1.103) small and using Lemma 7 to estimate $e_{0}$, we obtain

$$
\mathscr{E}(T ; K) \leq \epsilon \int_{0}^{T} \frac{1}{\bar{t}} \mathscr{E}(t ; K) d t+G(\epsilon) R \ln R \cdot \mathscr{G}\left(T ; \bar{\Psi}_{0}\right) .
$$

The final result follows from an application of Gronwall's inequality.

5.2. Estimates for $\tilde{e}$. As the boundary condition for $\tilde{e}$ has constant coefficients in time and the angular variable, we consider its Laplace transform in $t$ and Fourier transform in $\theta, \hat{e}_{n}$. Introducing differential operators

$$
\begin{gathered}
L_{n} \equiv \frac{\partial^{2}}{\partial r^{2}}+\frac{1}{r} \frac{\partial}{\partial r}-\frac{n^{2}}{r^{2}}-s^{2}, \quad D \equiv \frac{\partial}{\partial r}+s+\frac{1}{2 r} \equiv D^{*}+\frac{1}{2 r}, \\
C_{n} \equiv \frac{\left(4 n^{2}-1\right)}{8 R^{2}\left(s+\delta_{n}\right)},
\end{gathered}
$$

we have the problems $(n=1, \ldots, \infty)$ :

Problem 1.

$$
\begin{gathered}
L_{n} \hat{e}_{n}(r, s)=0, \quad 1 \leq r \leq R, \\
\hat{e}_{n}=0, r=1 ; \quad\left(D+C_{n}\right) \hat{e}_{n}=\hat{\Psi}_{n}, r=R .
\end{gathered}
$$


Our error estimates follow in three steps. We first note that the well-posedness of the problem for $\tilde{e}_{n}$ follows from general principles (e.g. [27, §3.3]). This implies the solution may be represented by its Laplace transform for $\Re s$ sufficiently large. Second, as the solution of a problem with analytic coefficients (with $s$ in the closed right half-plane) $\hat{e}_{n}$ must be a meromorphic function of $s$ if $\hat{\Psi}_{n}$ is. We will first prove that there are no poles in the closed right half-plane, which by the preceding comment implies that $\hat{e}_{n}$ is analytic. We then push the inversion contour to the imaginary $s$-axis, where we estimate the transform in terms of $\hat{\Psi}_{n}$. Finally, Plancherel's Theorem is used to give the final estimate.

To make the argument completely rigorous, one needs estimates for large $|s|$ in the right half-plane. Estimates of this type have been developed, for example, in [23]. We, however, have not carried out the details for our case.

Theorem 4. For $\Re s \geq 0$ there are no nontrivial solutions of Problem 1 with $\hat{\Psi}_{n}=0$.

Proof. Suppose $q$ is a solution of Problem 1 with zero data. Let $s=\xi+i \eta$, $\xi \geq 0$. Multiplying the equation by $\bar{q}$ and integrating from 1 to $R$ yields

$$
\begin{aligned}
I_{n} \equiv & \int_{1}^{R} r\left|\frac{\partial q}{\partial r}\right|^{2} d r+s^{2} \int_{1}^{R} r|q|^{2} d r \\
& +n^{2} \int_{1}^{R} \frac{1}{r}|q|^{2} d r+R\left(s+C_{n}(s)\right)|q(R)|^{2}=0 .
\end{aligned}
$$

We first note that

$$
\Re\left(s+C_{n}(s)\right)=\xi+\frac{4 n^{2}-1}{8 R^{2}} \cdot \frac{\xi+\delta_{n}}{\left(\xi+\delta_{n}\right)^{2}+\eta^{2}}>0,
$$

since $\delta_{n}>0$. Therefore,

$$
0=\Re I_{n} \geq\left(\xi^{2}-\eta^{2}+\frac{n^{2}}{R^{2}}\right) \int_{1}^{R} r|q|^{2} d r
$$

which implies

$$
\eta^{2} \geq \xi^{2}+\frac{n^{2}}{R^{2}}
$$

Looking now at the imaginary part,

$$
\Im I_{n}=2 \xi \eta \int_{1}^{R} r|q|^{2} d r+R \cdot \Im\left(s+C_{n}\right)|q(R)|^{2},
$$

we conclude that

$$
\frac{\Im\left(s+C_{n}\right)}{\eta} \leq 0 .
$$

Writing this out and using (5.2.118), we reach a contradiction:

$$
\frac{\Im\left(s+C_{n}\right)}{\eta}=1-\frac{4 n^{2}-1}{8 R^{2}} \cdot \frac{1}{\left(\xi+\delta_{n}\right)^{2}+\eta^{2}} \geq 1-\frac{4-n^{-2}}{8} \geq \frac{1}{2}
$$

This completes the proof. 
We now restrict attention to the imaginary $s$-axis, where we have the identity due to Morawetz and Ludwig [23]:

$$
\begin{aligned}
2 \Re(r \overline{D w} \cdot L w)= & \nabla \cdot\left(2 \Re(r \overline{D w} \nabla w)-\left(s^{2}|w|^{2}+|\nabla w|^{2}\right) \vec{x}\right) \\
& -\left(|\nabla w|^{2}-\left|\frac{\partial w}{\partial r}\right|^{2}\right)-\left|D^{*} w\right|^{2} .
\end{aligned}
$$

Integrating this (with $w=\hat{e}_{n}$ ) and using the definition of $D$ and the boundary condition at $r=1$, we obtain

$$
\begin{aligned}
& \int_{1}^{R}\left(\left|D^{*} \hat{e}_{n}\right|^{2}+\frac{n^{2}}{r^{2}}\left|\hat{e}_{n}\right|^{2}\right) r d r \\
& =\left.\left(2 \Re\left(r \overline{D \hat{e}_{n}} \frac{\partial \hat{e}_{n}}{\partial r}\right)-r\left(\left(s^{2}+\frac{n^{2}}{r^{2}}\right)\left|\hat{e}_{n}\right|^{2}+\left|\frac{\partial \hat{e}_{n}}{\partial r}\right|^{2}\right)\right)\right|_{1} ^{R} \\
& =\left.R^{2}\left(\left|D \hat{e}_{n}\right|^{2}-\frac{1+4 n^{2}}{4 R^{2}}\left|\hat{e}_{n}\right|^{2}\right)\right|_{r=R}-\left|\frac{\partial \hat{e}_{n}}{\partial r}(1)\right|^{2} .
\end{aligned}
$$

We now use the boundary condition to estimate the boundary term at $r=R$. We have the inequality

$$
\left|C_{n}\right| \leq \frac{2 n-1}{2 R},
$$

which implies, for any $\mu>0$,

$$
\left|D \hat{e}_{n}\right|^{2} \leq(1+\mu) \frac{4 n^{2}-4 n+1}{4 R^{2}}\left|\hat{e}_{n}\right|^{2}+(1+(4 / \mu)) \hat{\Psi}_{n}^{2} .
$$

We can use this inequality to estimate the boundary terms in (5.2.123), which leads to the following lemma.

Lemma 8. At $r=R$ we have

$$
\left(\left|D \hat{e}_{n}\right|^{2}-\frac{1+4 n^{2}}{4 R^{2}}\left|\hat{e}_{n}\right|^{2}\right)+\frac{3}{4 R^{2}}\left|\hat{e}_{n}\right|^{2} \leq 5 n\left|\hat{\Psi}_{n}\right|^{2} .
$$

Proof. The lemma is a direct consequence of (5.2.125) with $\mu=\frac{1}{n}$.

Substituting into (5.2.123) and applying Plancherel's Theorem, we have

Lemma 9. For any $T \geq 0$ there holds

$$
\begin{aligned}
& \int_{0}^{T} \int_{1}^{R}\left(\frac{n^{2}}{r^{2}}\left|e_{n}\right|^{2}+\left|\frac{\partial e_{n}}{\partial r}+\frac{\partial e_{n}}{\partial t}\right|^{2}\right) r d r d t+\frac{3}{4} \int_{0}^{T}\left|e_{n}(R)\right|^{2} d t \\
& \quad \leq 5 R^{2} n \int_{0}^{T}\left|\bar{\Psi}_{n}\right|^{2} d t .
\end{aligned}
$$

Noting that $\tilde{e}$ has zero average in $\theta$, we see that Lemma 9 yields a bound on $\|\tilde{e}\|$. (Here, $\|\cdot\|$ denotes the $L_{2}$ norm on $\Upsilon$.) However, it cannot be used 
directly to get a pointwise bound. For that, we go back to the standard energy equality (again taking account of the zero Dirichlet data at $r=1$ ):

$$
\begin{aligned}
\frac{1}{2} \int_{1}^{R} & \left(\left|\frac{\partial e_{n}}{\partial r}\right|^{2}+\left|\frac{\partial e_{n}}{\partial t}\right|^{2}+\frac{n^{2}}{r^{2}}\left|e_{n}\right|^{2}\right) r d r \\
& =R \cdot \Re\left(\int_{0}^{T} \frac{\partial e_{n}}{\partial r}(R, t) \frac{\partial e_{n}}{\partial t}(R, t) d t\right) .
\end{aligned}
$$

The untransformed boundary condition is

$$
\frac{\partial e_{n}}{\partial r}=-\frac{\partial e_{n}}{\partial t}+\Gamma_{n}(t)
$$

where

$$
\Gamma_{n}(t)=-\frac{1}{2 R} e_{n}(R, t)-\frac{4 n^{2}-1}{8 R^{2}} \int_{0}^{t} e^{-\delta_{n}(t-p)} e_{n}(R, p) d p+\bar{\Psi}_{n}(t) .
$$

We first estimate the energy in terms of $\Gamma_{n}$ by substituting the boundary condition into (5.2.128), applying the elementary product inequality:

$$
\begin{aligned}
& \frac{1}{2} \int_{1}^{R}\left(\left|\frac{\partial e_{n}}{\partial r}\right|^{2}+\left|\frac{\partial e_{n}}{\partial t}\right|^{2}+\frac{n^{2}}{r^{2}}\left|e_{n}\right|^{2}\right) r d r+\frac{R}{2} \int_{0}^{T}\left|\frac{\partial e_{n}}{\partial t}\right|^{2} d t \\
& \quad \leq \frac{R}{2} \int_{0}^{T}\left|\Gamma_{n}\right|^{2} d t .
\end{aligned}
$$

We now estimate $\Gamma_{n}$, using standard estimates for convolutions:

$$
\begin{aligned}
& \int_{0}^{T}\left|\Gamma_{n}\right|^{2} d t \\
& \quad \leq 3\left(\frac{1}{4 R^{2}} \int_{0}^{T}\left|e_{n}\right|^{2} d t+\frac{\left(4 n^{2}-1\right)^{2}}{64 R^{4}} \int_{0}^{T}\left|\int_{0}^{t} e^{-\delta_{n}(t-p)} e_{n}(R, p) d p\right|^{2} d t\right. \\
& \left.\quad \leq \frac{3 n^{2}}{R^{2}} \int_{0}^{T}\left|e_{n}\right|^{2} d t+\int_{0}^{T}\left|\bar{\Psi}_{n}\right|^{2} d t\right)
\end{aligned}
$$

Combining these leads to an estimate which does not involve the solution at the boundary. We introduce the fractional Sobolev norm at the boundary, $\|w\|_{3 / 2, R} \equiv\left(R \sum_{n=1}^{\infty} n^{3}\left|w_{n}\right|^{2}\right)^{1 / 2}$, and let $\|\cdot\|$ denote the $L_{2}$ norm in $\Upsilon$.

Theorem 5. For smooth errors $\tilde{e}$, any $T>0$, and an $O(1)$ constant $M$, we have the estimate 


$$
\begin{aligned}
& \left\|\frac{\partial \tilde{e}}{\partial r}(\cdot, T)\right\|^{2}+\left\|\frac{\partial \tilde{e}}{\partial t}(\cdot, T)\right\|^{2}+\left\|\frac{1}{r} \frac{\partial \tilde{e}}{\partial \theta}(\cdot, T)\right\|^{2} \\
& \quad+R \int_{0}^{T}\left(\frac{\partial \tilde{e}}{\partial t}(R, t)\right)^{2} d t+\frac{8}{R} \int_{0}^{T}\left\|\frac{1}{r} \frac{\partial^{2} \tilde{e}}{\partial \theta^{2}}(\cdot, t)\right\|^{2} d t \\
& \quad \leq M \int_{0}^{T}\|\tilde{\Psi}(t)\|_{3 / 2, R}^{2} d t .
\end{aligned}
$$

Proof. We begin by multiplying the inequality in Lemma 9 by $\frac{4 n^{2}}{R}$ and adding the result to (5.2.131). Then, applying (5.2.132), we deduce

$$
\begin{gathered}
\int_{1}^{R}\left(\left|\frac{\partial e_{n}}{\partial r}\right|^{2}+\left|\frac{\partial e_{n}}{\partial t}\right|^{2}+\frac{n^{2}}{r^{2}}\left|e_{n}\right|^{2}\right) r d r+\frac{R}{2} \int_{0}^{T}\left|\frac{\partial e_{n}}{\partial t}\right|^{2} d t \\
+\frac{4}{R} \int_{0}^{T} \int_{1}^{R} \frac{n^{4}}{r^{2}}\left|e_{n}\right|^{2} r d r d t+\frac{3 n^{2}}{R} \int_{0}^{T}\left|e_{n}(R)\right|^{2} d t \\
\leq \frac{3 n^{2}}{R} \int_{0}^{T}\left|e_{n}(R)\right|^{2} d t+M R n^{3} \int_{0}^{T}\left|\bar{\Psi}_{n}\right|^{2} d t .
\end{gathered}
$$

Subtracting the term involving $e_{n}(R)$ from both sides and applying Plancherel's Theorem yields the desired result.

We can now estimate the pointwise error using a Poincaré inequality:

Theorem 6. There holds

$$
\tilde{e}^{2}(r, \theta, T) \leq M \ln r \int_{0}^{T}\|\tilde{\Psi}(t)\|_{3 / 2, R}^{2} d t .
$$

Proof. Combining (5.1.105) (with $R$ replaced by $r$ ) with the preceding theorem immediately yields the desired result.

5.3. Asymptotics of $\Psi$ and $e$. We now use asymptotic expansions of the solution of the wave equation in combination with the energy estimates above to derive asymptotic error estimates. We begin with the well-known progressive wave (Friedlander) expansion, valid for $((t-r) / r) \ll 1$ :

$$
u \sim \sum_{j} \frac{f_{j}(t-r, \theta)}{r^{(2 j+1) / 2}},
$$

where the functions $f_{j}(p, \theta)$ are related by

$$
2 j \frac{\partial f_{j}}{\partial p}=-\frac{\partial^{2} f_{j-1}}{\partial \theta^{2}}-(j-(1 / 2))^{2} f_{j-1}, \quad j=1,2, \ldots .
$$

It is worth noting that Friedlander [9] only proves the validity of the analogous expansion in three space dimensions. Karp [20] directly analyzes the two-dimensional expansion in the frequency domain. See also [15] for a timedomain approach based on the Riemann function.

All $f_{j}$ 's are determined by the radiation field, $f_{0}$. Expanding in a Fourier series in $\theta, f_{j}=\sum_{n} f_{j n}(p) e^{i n \theta}$, and applying the boundary condition (3.0.56), 
we obtain, using (5.3.137),

$$
(B u)_{n} \sim B_{n}\left(\sum_{j} \frac{f_{j n}(t-r)}{r^{(2 j+1) / 2}}\right)=O\left(r^{-\frac{7}{2}}\right) .
$$

This implies, if $t=R+O(1)$, that

$$
\bar{\Psi}_{n}=O\left(R^{-\frac{7}{2}}\right) .
$$

The short-time behavior of the error can then be extracted by mimicking the scaling arguments of [3]. This yields the following result:

Theorem 7. For $0 \leq t \leq(R+T)$, $T$ fixed, we have

$$
\|e\|_{1, \Upsilon}=O\left(R^{-2}\right), \quad R \rightarrow \infty .
$$

(Here, $\|\cdot\|_{1, \Upsilon}$ is the space-time Sobolev norm involving derivatives up to order 1.)

Estimates of the time-integral of the error can then be obtained using a Poincaré inequality:

$$
\int_{0}^{R+T} e^{2}(r, \theta, t) d t=O\left(\ln r \cdot R^{-4}\right), \quad R \rightarrow \infty, \quad T \text { fixed. }
$$

The progressive wave expansion, however, breaks down as $t \rightarrow \infty$. Therefore, to analyze the long-time behavior of the solution, we must use the longtime expansion of Muravei [25, §5]. We decompose the solution $u=u_{0}+\tilde{u}$ in the same way as we decomposed the error, and assume the data approaches its limit at least as fast as $O\left(t^{-2}\right)$. Then we have

$$
\begin{gathered}
u_{0}=u_{0, \infty}\left(1-\frac{\ln r}{\ln 2 t}\right)+O\left(\frac{\ln r}{\ln ^{3} t}\right), t \rightarrow \infty, \\
\tilde{u}=\tilde{u}_{\infty}(r, \theta)+O\left(\ln t \cdot t^{-2}\right), \quad t \rightarrow \infty
\end{gathered}
$$

Substituting these expressions into (3.0.56), we obtain

$$
\Psi_{0}=O\left(\frac{\ln R}{R^{2} \ln ^{3} t}\right)
$$

$$
\bar{\Psi}_{0}=\int_{0}^{t} e^{-\int_{\tau}^{t} \delta_{0}(\eta) d \eta} \Psi_{0}(\tau) d \tau \sim \frac{\Psi_{0}(t)}{\delta_{0}(t)}=O\left(\frac{\ln R}{R \ln ^{3} t}\right),
$$

for the mean and, using the fact that $\frac{\partial u_{n, \infty}}{\partial r}+\frac{n}{R} u_{n, \infty}=0$,

$$
\tilde{\Psi}=O\left(\ln t \cdot t^{-3}\right), \quad \stackrel{\tilde{\Psi}}{\Psi}=O\left(\ln t \cdot t^{-3}\right) .
$$

We note that these are smooth functions, so that the estimates can be extended 
to norms involving derivatives. Substituting these into the energy inequalities yields the following error estimates:

Theorem 8. Suppose the conditions of Theorem 3 hold and that the data approaches its $t \rightarrow \infty$ limit at least as fast as $O\left(t^{-2}\right)$. Then for some constant $L$ independent of $R$, and $T$ sufficiently large, we have

$$
\mathscr{E}(T ; K)<L\left(\frac{\bar{T}^{2} \ln ^{3} R}{R \ln ^{6} T}\right) .
$$

Proof. Substituting (5.3.145) into (5.1.103) and using the additional fact that $\frac{\partial \Psi_{0}}{\partial t}=O\left(t^{-1} \ln ^{-4} t\right)$, we obtain ( $L$ is a generic $R, T$-independent constant)

$$
\begin{gathered}
\mathscr{G}<L \frac{\bar{T}^{2} \ln ^{2} R}{R^{2} \ln ^{6} T}, \\
\frac{d \mathscr{G}}{d t}<L \frac{\bar{T} \ln ^{2} R}{R^{2} \ln ^{6} T}, \\
\int_{0}^{T} \bar{t}^{-\epsilon} \frac{d \mathscr{G}}{d t}<L \frac{\bar{T}^{2-\epsilon} \ln ^{2} R}{R^{2} \ln ^{6} T} .
\end{gathered}
$$

Combining this inequality with Theorem 3 yields the desired result.

Lemma 7 can be used to estimate the pointwise error:

Corollary 1. Suppose the hypotheses of Theorem 8 hold. Then

$$
e_{0}^{2}(r, T) \leq L \frac{\ln ^{3} R \ln r}{R \ln ^{6} T} .
$$

Remark. The computations were made with $R$ too small to directly verify the dependence of the error on $R$. In particular, the function $\left(\ln ^{3} R\right) / R$ is not monotonically decreasing unless $R$ is sufficiently large. Therefore, it is unlikely that this estimate displays the correct $R$ behavior for small to moderate $R$.

Finally, combining Theorem 6 with (5.3.146), we see that $\tilde{e}$ decays more rapidly in time:

Theorem 9. As $T \rightarrow \infty$, there holds

$$
\tilde{e}^{2}(r, \theta, T) \leq \ln r \cdot O\left(\frac{\ln ^{2} T}{T^{5}}\right) .
$$

\section{BIBLIOGRAPHY}

1. M. Abramowitz and I. A. Stegun, Handbook of mathematical functions, Dover, New York, 1970.

2. A. Barry, J. Bielak, and R. MacCamy, On absorbing boundary conditions for wave propagation, J. Comput. Phys. 79 (1988), 449-468.

3. A. Bayliss and E. Turkel, Radiation boundary conditions for wave-like equations, Comm. Pure Appl. Math. 33 (1980), 707-725. 
4. J. Bielak and R. MacCamy, Dissipative boundary conditions for one-dimensional wave propagation, J. Integral Equations Appl. 2 (1990), 307-331.

5. Y.-M. Chen, The transient behavior of diffraction of a plane pulse by a circular cylinder, Internat. J. Engrg. Sci. 2 (1964), 417-429.

6. B. Engquist and A. Majda, Absorbing boundary conditions for the numerical simulation of waves, Math. Comp. 31 (1977), 629-651.

7. B. Engquist and L. Halpern, Far field boundary conditions for computation over long time, Appl. Numer. Math. 4 (1988), 21-45.

8. _ Long time behavior of absorbing boundary conditions, Math. Methods Appl. Sci. 13 (1990), 189-204.

9. F.G. Friedlander, On the radiation field of pulse solutions of the wave equation, Proc. Roy. Soc. Ser. A 279 (1964), 386-394.

10. D. Givoli, Non-reflecting boundary conditions: A review, J. Comput. Phys. 94 (1991), 1-29.

11. G. Gripenberg, S.-O. Londen, and O. Staffans, Volterra integral and functional equations, Cambridge Univ. Press, Cambridge, 1990.

12. B. Gustafsson, Inhomogeneous conditions at open boundaries for wave propagation problems, Appl. Numer. Math. 4 (1988), 3-19.

13. T. Ha-Duong and P. Joly, On the stability analysis of boundary conditions for the wave equation by energy methods, Part I: the homogeneous case, INRIA Rapports de Recherche 1306, (1990).

14. T. Hagstrom, Consistency and convergence for numerical radiation conditions, Mathematical and Numerical Aspects of Wave Propagation Phenomena (G. Cohen, L. Halpern, and P. Joly, eds.), SIAM, Philadelphia, PA, 1991, pp. 283-292.

15. T. Hagstrom and S.I. Hariharan, Far field expansion for anisotropic wave equations, Computational Acoustics: Scattering, Gaussian Beams and Aeroacoustics, vol. 2 (D. Lee, A. Cakmak, and R. Vichnevetsky, eds.), North-Holland, Amsterdam, 1990, pp. 283-294.

16. T. Hagstrom and H.B. Keller, Exact boundary conditions at an artificial boundary for partial differential equations in cylinders, SIAM J. Math. Anal. 17 (1986), 322-341.

17. L. Halpern and J. Rauch, Error analysis for absorbing boundary conditions, Numer. Math. 51 (1987), 459-467.

18. S.I. Hariharan and R. MacCamy, Low frequency acoustic and electromagnetic scattering, Appl. Numer. Math. 2 (1986), 29-35.

19. R. Higdon, Absorbing boundary conditions for difference approximations to the multidimensional wave equation, Math. Comp. 47 (1986), 437-460.

20. S. Karp, A convergent 'far-field' expansion for two-dimensional radiation functions, Comm. Pure Appl. Math. 14 (1961), 427-434.

21. J. Keller and D. Givoli, Exact non-reflecting boundary conditions, J. Comput. Phys. 82 (1989), 172-192.

22. P. D. Lax, C. S. Morawetz, and R. S. Phillips, Exponential decay of solutions of the wave equation in the exterior of a star-shaped obstacle, Comm. Pure Appl. Math. 16 (1963), 477-486.

23. C. S. Morawetz and D. Ludwig, An inequality for the reduced wave operator and the justification of geometrical optics, Comm. Pure Appl. Math. 21 (1968), 187-203.

24. V.P. Mihailov, On the principle of limiting amplitude, Soviet Math. Dokl. 5 (1964), 15991602.

25. L. Muravei, On the asymptotic behavior, for large values of the time, of solutions of exterior boundary value problems for the wave equation with two space variables, Math. USSR-Sb. 35 (1979), 377-423.

26. The wave equation and the Helmholtz equation in an unbounded domain with a star-shaped boundary, Proc. Steklov Inst. Math. 185 (1990), 191-201.

27. R. Sakamoto, Hyperbolic boundary value problems, Cambridge Univ. Press, Cambridge, 1982. 
28. V. Vladimirov, Yu. Drozhzhinov, and B. Zav'yalov, Tauberian theorems for generalized functions, Kluwer, Dordrecht, 1988.

29. D. Widder, The Laplace transform, Princeton Univ. Press, Princeton, NJ, 1946.

Department of Mathematics and Statistics, The University of New Mexico, AlbuQUERQUE, NEW MEXICO 87131

E-mail address: hagstrom@mimir.unm.edu

Department of Mathematical Sciences, University of Akron, Akron, Ohio 44325

E-mail address: hari@gauss math.uakron.edu

Department of Mathematics, Carnegie-Mellon University, Pittsburgh, Pennsylvania 15213 


\title{
Supplement to
}

\section{ON THE ACCURATE LONG-TIME SOLUTION OF THE WAVE EQUATION IN EXTERIOR DOMAINS: ASYMPTOTIC EXPANSIONS AND CORRECTED BOUNDARY CONDITIONS}

\author{
THOMAS HAGSTROM, S. I. HARIHARAN, AND R. C. MACCAMY
}

\section{Appendix A. Construction and analysis of H}

We seek a function, $\mathcal{H}(\tau)$, whose Laplace transform, $\hat{\mathcal{H}}(s)=(s \ln s)^{-1}+O(1)$ as $s \rightarrow 0$, has no other singularities for $\Re s \geq 0$ and decays as fast as $(s \ln s)^{-1}$ as $s \rightarrow \infty$. We make use of the following formula, which may be found in most tables of Laplace transforms or verified by direct computation

$$
\mathcal{L}\left(\int_{0}^{\infty} \frac{\tau^{u-1+\alpha}}{\Gamma(u+\alpha)} d u\right)=\frac{1}{s^{\alpha} \ln s}, \alpha \geq 0 .
$$

Let

$$
F(\tau)=\int_{0}^{1} \frac{\tau^{u-1}}{\Gamma(u)} d u
$$

so that

$$
\hat{F}(s)=\frac{1}{\ln s}-\frac{1}{s \ln s} .
$$

Define $\mathcal{H}(\tau)$ to be the unique decaying solution of

$$
\frac{d \mathcal{H}}{d \tau}-\mathcal{H}=F
$$

It may be represented in integral form as

$$
\mathcal{H}(\tau)=-\int_{\tau}^{\infty} e^{\tau-p}\left(\int_{0}^{1} \frac{p^{u-1}}{\Gamma(u)} d u\right) d p .
$$

To compute the large $\tau$ asymptotics of $\mathcal{H}$, we first compute the large $p$ asymptotics of $F(p)$. By Watson's lemma we find

$$
F(p)=\frac{1}{\ln p}-\frac{\gamma}{(\ln p)^{2}}+O\left((\ln p)^{-3}\right) .
$$

Substituting this into the expression for $\mathcal{H}$ and integrating by parts, we finally have

$$
\mathcal{H}(\tau)=-\frac{1}{\ln \tau}+\frac{\gamma}{(\ln \tau)^{2}}+O\left((\ln \tau)^{-3}\right), \quad \tau \rightarrow \infty .
$$

\section{Appendix B. Generalizations to Noncircular Boundaries}

In this section we consider general artificial boundaries, concentrating on the construction of the low-frequency expansion of the exact condition. Our purpose is twofold. First, we want to show that the lower bounds on error decay for local in time constant-coefficient conditions cannot be improved by changing the artificial boundary shape. Second, we want to indicate how to construct uniformly accurate conditions for such boundaries. (This may be useful for scattering by bodies with large aspect ratio.) Similar ideas may be applied in three space dimensions. The construction of the low-frequency expansion is discussed in more detail in [18]. 


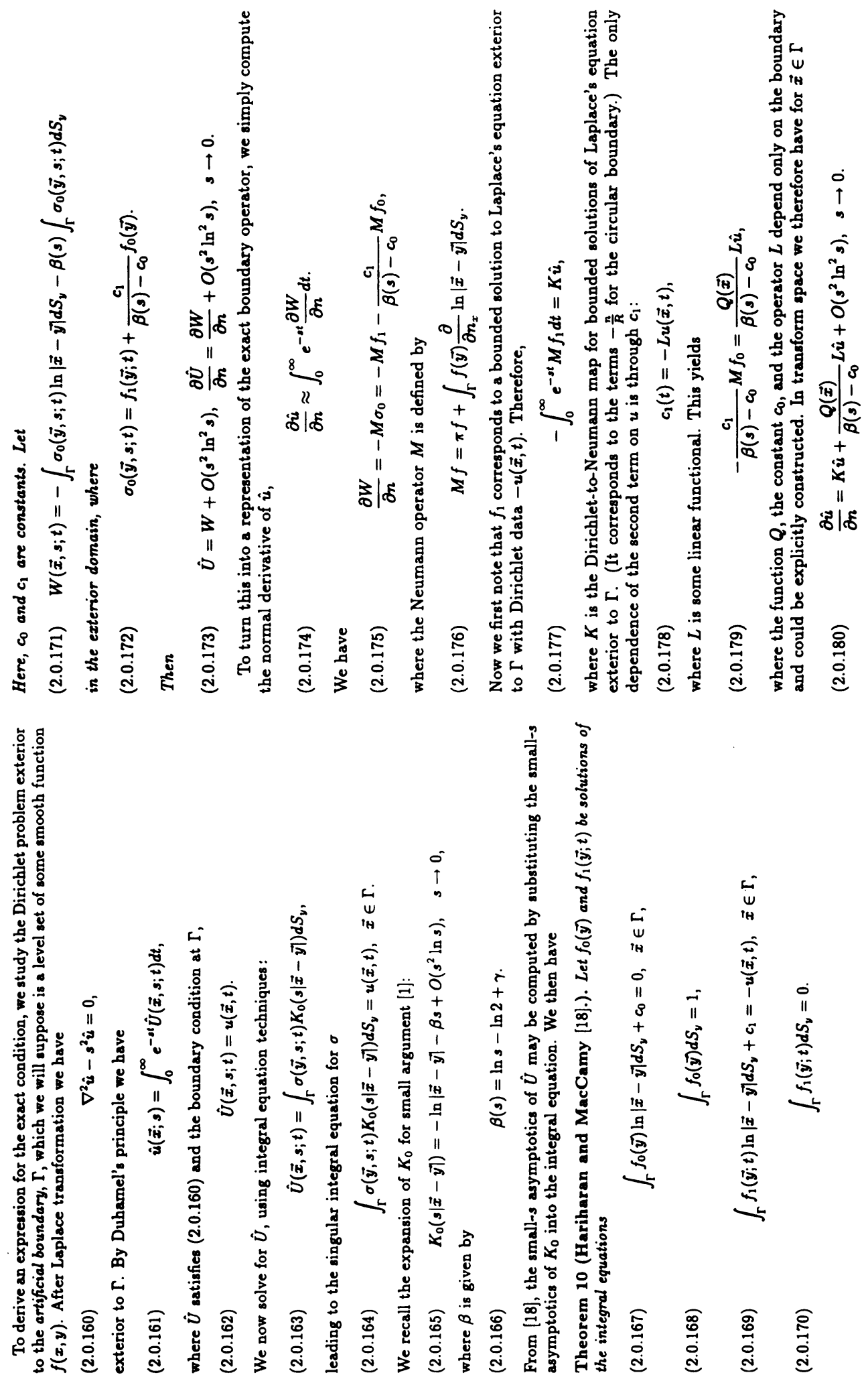



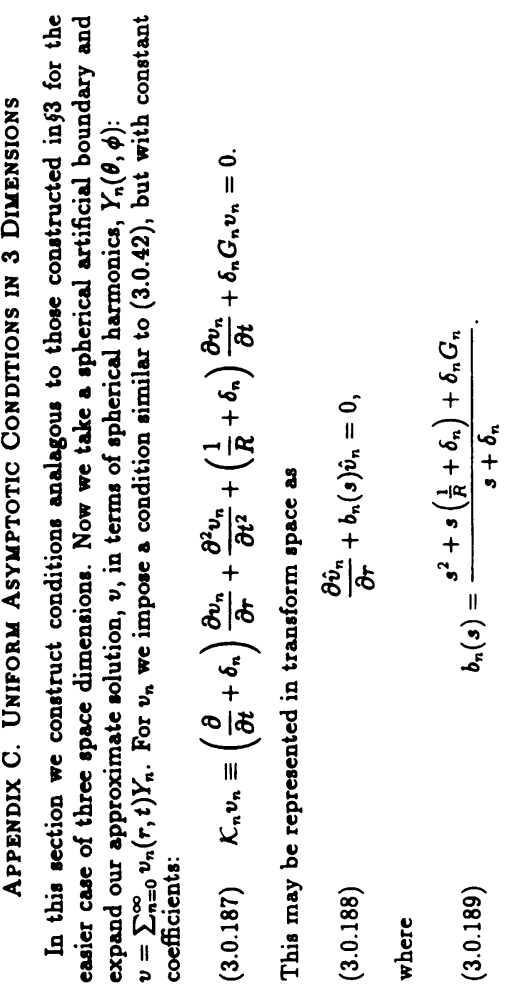

蒙

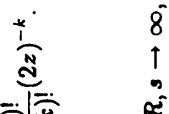

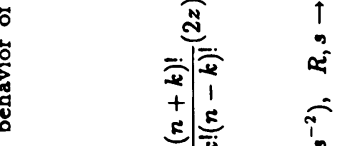

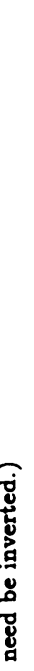

商

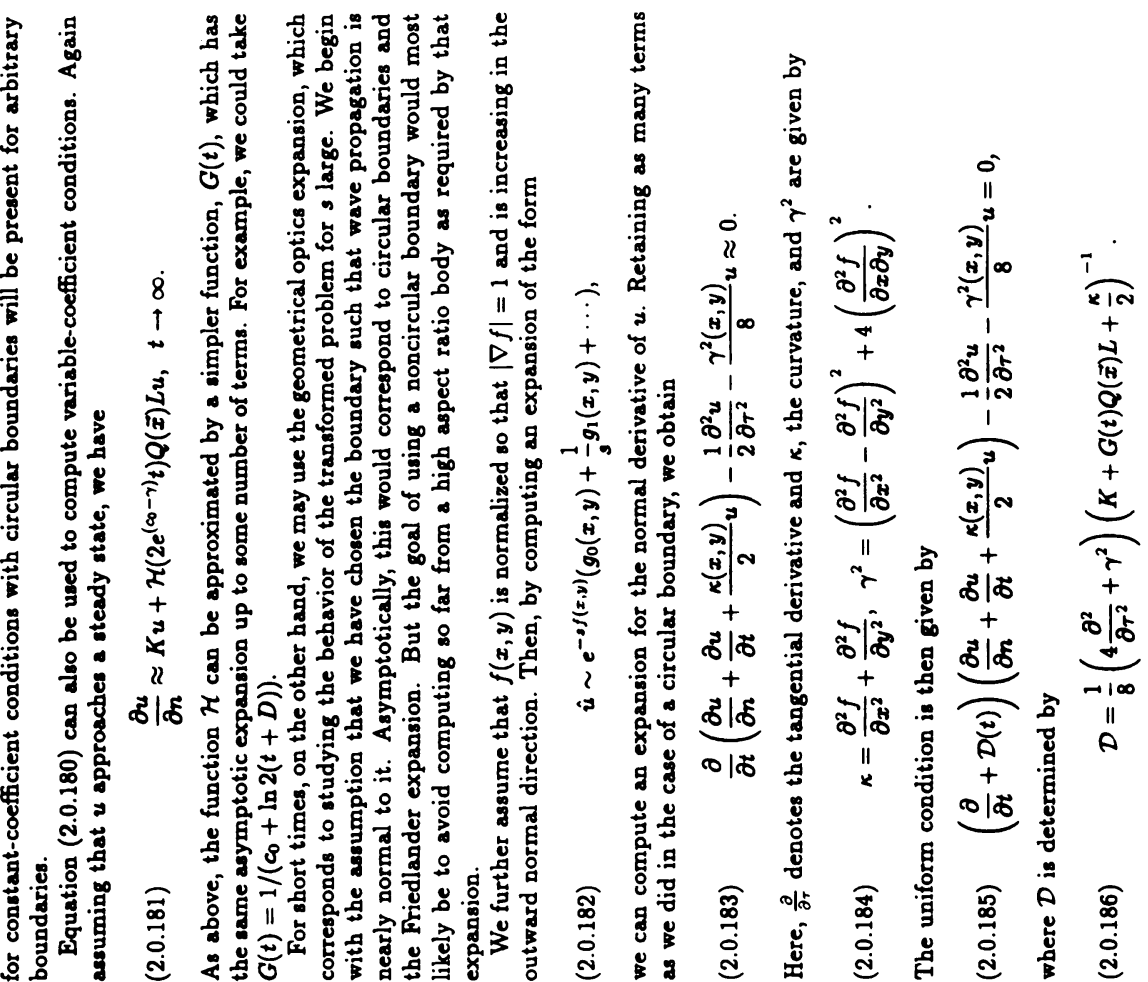




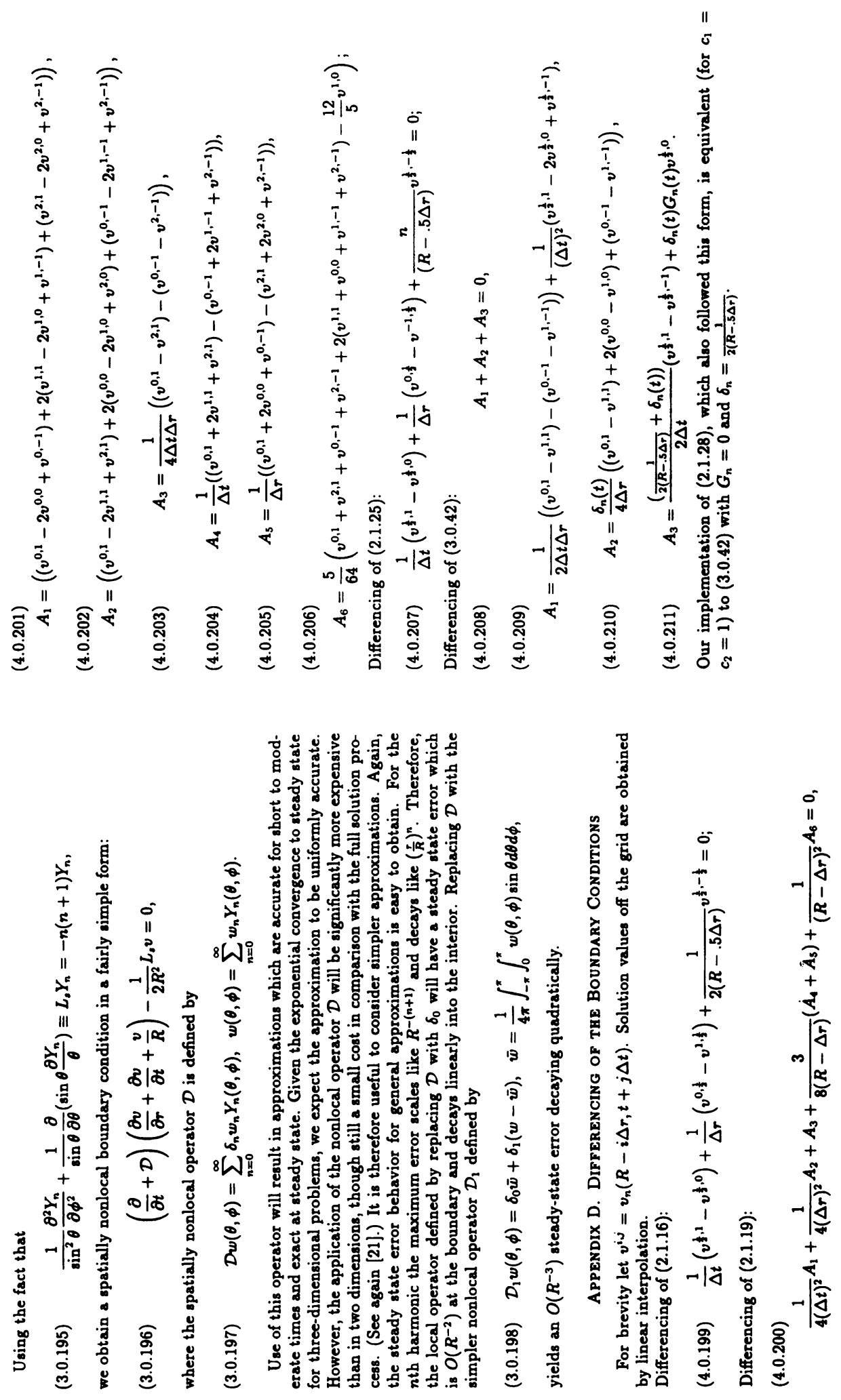

